\title{
14. GRAIN SIZE ANALYSIS, LEG 3
}

\author{
A. C. Pimm, Deep Sea Drilling Project, East Coast Repository, \\ Lamont-Doherty Geological Observatory, Palisades, New York
}

\section{INTRODUCTION}

The grain size results from Leg 3 were almost entirely a reflection of the microfossil content of the sediments. Wherever the sand fraction makes up more than about 1 per cent of the sediment, then it is comprised of planktonic foraminifera with a few benthonics, or in a few places Radiolaria. Similarly, the silt fraction usually reflects the nannoplankton content of the sediment. Where the sediment is comprised almost entirely of clay it is usually zeolitic red clay with practically no fossils.

The complete grain size results are given as Table 1 , and a summary of the nature of the coarse fraction is given as Table 2. Comments on the grain size results from each drill hole are given below.

\section{RESULTS}

\section{Hole 13 and 13A}

Hole 13 mainly contains clay in its upper part, but further down there are increasing amounts of sand and silt. In the lower part the sediment falls in the sand-silt-clay or silty clay grade. The clays in Cores 1 and 2 are zeolitic with only small amounts of sand (foraminifera and Radiolaria) and some silt (nannoplankton). The increase in the sand fraction in Core 3 is due to an abundance of Radiolaria.

Sediments in Hole 13A are either silty clay or clay. Core 1 is a silty clay with 17 per cent sand (Radiolaria). Cores 2 and 3 are clay; however, they were described in the hole summaries as nannoplankton chalk oozes. On grain size and carbonate content results these sediments are more probably zeolitic clays (check X-ray results). Core 4 is a nannofossil chalk ooze with nannofossils making up 22 per cent silt and foraminifera 15 per cent sand. Cores 5 and 7 contain over 80 per cent clay.

\section{Hole 14}

The sediments from Hole 14 are mainly clayey silt with some silty clay. The sediments are partly zeolitic

TABLE 1

Leg 3 Grain Size Results

\begin{tabular}{ccccccll}
\hline Hole & Core & Section & $\begin{array}{c}\text { Sampled } \\
\text { at }(\mathrm{cm})\end{array}$ & $\begin{array}{c}\text { Sand } \\
(\%)\end{array}$ & $\begin{array}{c}\text { Silt } \\
(\%)\end{array}$ & $\begin{array}{l}\text { Clay } \\
(\%)\end{array}$ & Classification \\
\hline 13 & 1 & 1 & $7-9$ & 8.1 & 16.7 & 75.2 & Clay \\
13 & 1 & 2 & $4-6$ & 10.2 & 15.6 & 74.2 & Silty clay \\
13 & 1 & 3 & $4-6$ & 6.4 & 17.4 & 76.2 & Clay \\
13 & 1 & 4 & $7-9$ & 4.5 & 19.6 & 75.9 & Clay \\
13 & 1 & 5 & $8-10$ & 3.4 & 20.1 & 76.5 & Clay \\
13 & 2 & 1 & $32-34$ & 4.3 & 20.5 & 75.2 & Clay \\
13 & 2 & 2 & $8-10$ & 1.6 & 19.4 & 79.0 & Clay \\
13 & 2 & 3 & $4-6$ & 2.2 & 23.2 & 74.6 & Silty clay \\
13 & 2 & 4 & $2-4$ & 0.1 & 8.9 & 91.0 & Clay \\
13 & 2 & 5 & $2-4$ & 0.2 & 8.5 & 91.3 & Clay \\
13 & 2 & 5 & $74-76$ & 25.9 & 27.2 & 46.9 & Sand silt clay \\
13 & 2 & 6 & $2-4$ & 0.2 & 8.3 & 91.5 & Clay \\
13 & 3 & 1 & $80-82$ & 26.7 & 28.0 & 45.3 & Sand silt clay \\
\hline
\end{tabular}


TABLE 1 - Continued

\begin{tabular}{|c|c|c|c|c|c|c|c|}
\hline Hole & Core & Section & $\begin{array}{l}\text { Sampled } \\
\text { at }(\mathrm{cm})\end{array}$ & $\begin{array}{c}\text { Sand } \\
(\%)\end{array}$ & $\begin{array}{l}\text { Silt } \\
(\%)\end{array}$ & $\begin{array}{l}\text { Clay } \\
(\%)\end{array}$ & Classification \\
\hline 13 & 3 & 2 & $5-7$ & 24.9 & 23.9 & 51.2 & Sand silt clay \\
\hline 13 & 3 & 3 & $4-6$ & 19.6 & 26.6 & 53.8 & Silty clay \\
\hline 13 & 3 & 4 & $1-3$ & 24.5 & 28.0 & 47.5 & Sand silt clay \\
\hline 13 & 3 & 5 & $2-4$ & 17.9 & 21.7 & 60.4 & Silty clay \\
\hline 13 & 3 & 6 & $3-5$ & 14.9 & 23.6 & 61.5 & Silty clay \\
\hline $13 \mathrm{~A}$ & 1 & 1 & $66-68$ & 17.0 & 25.3 & 57.7 & Silty clay \\
\hline $13 \mathrm{~A}$ & 2 & 1 & $103-105$ & 0.3 & 13.1 & 86.6 & Clay \\
\hline $13 \mathrm{~A}$ & 3 & 1 & $81-83$ & 0.2 & 7.6 & 92.2 & Clay \\
\hline $13 \mathrm{~A}$ & 3 & 2 & $2-4$ & 0.0 & 6.4 & 93.6 & Clay \\
\hline $13 \mathrm{~A}$ & 3 & 3 & $2-4$ & 0.0 & 6.9 & 93.1 & Clay \\
\hline $13 \mathrm{~A}$ & 3 & 4 & $3-5$ & 0.5 & 9.5 & 90.0 & Clay \\
\hline $13 \mathrm{~A}$ & 4 & 1 & $35-37$ & 15.2 & 21.7 & 63.1 & Silty clay \\
\hline $13 \mathrm{~A}$ & 5 & 1 & $17-19$ & 3.8 & 14.8 & 81.4 & Clay \\
\hline $13 \mathrm{~A}$ & 7 & 1 & $10-12$ & 1.1 & 15.8 & 83.1 & Clay \\
\hline 14 & $1 \mathrm{~A}$ & 1 & $52-54$ & 0.3 & 47.8 & 51.9 & Silty clay \\
\hline 14 & $1 \mathrm{~A}$ & 2 & $10-12$ & 0.5 & 55.6 & 43.9 & Clayey silt \\
\hline 14 & $1 \mathrm{~A}$ & 3 & $2-4$ & 0.2 & 46.5 & 53.3 & Silty clay \\
\hline 14 & $1 \mathrm{~A}$ & 4 & $22-24$ & 0.1 & 47.2 & 52.7 & Silty clay \\
\hline 14 & $1 \mathrm{~A}$ & 5 & $2-4$ & 0.7 & $43.2^{\prime}$ & 56.1 & Silty clay \\
\hline 14 & $1 \mathrm{~A}$ & 6 & $4-6$ & 0.5 & 53.3 & 46.2 & Clayey silt \\
\hline 14 & 2 & 1 & $3-5$ & 7.0 & 48.9 & 44.1 & Clayey silt \\
\hline 14 & 2 & 2 & $2-4$ & 6.4 & 47.5 & 46.1 & Clayey silt \\
\hline 14 & 2 & 3 & $2-4$ & 3.1 & 47.2 & 49.7 & Silty clay \\
\hline 14 & 2 & 4 & $2-4$ & 4.9 & 48.0 & 47.1 & Clayey silt \\
\hline 14 & 2 & 5 & $3-5$ & 4.7 & 50.7 & 44.6 & Clayey silt \\
\hline 14 & 3 & 1 & $2-4$ & 1.2 & 54.6 & 44.2 & Clayey silt \\
\hline 14 & 3 & 2 & $2-4$ & 1.1 & 55.8 & 43.1 & Clayey silt \\
\hline 14 & 3 & 3 & $2-4$ & 0.6 & 58.2 & 41.2 & Clayey silt \\
\hline 14 & 3 & 4 & $2-4$ & 1.1 & 57.4 & 41.5 & Clayey silt \\
\hline 14 & 3 & 5 & $2-4$ & 1.1 & 60.5 & 38.4 & Clayey silt \\
\hline 14 & 3 & 6 & $6-8$ & 0.9 & 58.0 & 41.1 & Clayey silt \\
\hline 14 & 4 & 1 & $35-37$ & 0.0 & 54.1 & 45.9 & Clayey silt \\
\hline 14 & 5 & 1 & $4-6$ & 0.6 & 46.0 & 53.4 & Silty clay \\
\hline 14 & 5 & 2 & $2-4$ & 0.7 & 48.3 & 51.0 & Silty clay \\
\hline 14 & 5 & 3 & $6-8$ & 0.7 & 48.4 & 50.9 & Silty clay \\
\hline 14 & 5 & 4 & $5-7$ & 0.2 & 42.1 & 57.7 & Silty clay \\
\hline 14 & 5 & 6 & $2-4$ & 0.7 & 49.3 & 50.0 & Silty clay \\
\hline
\end{tabular}




\begin{tabular}{|c|c|c|c|c|c|c|c|}
\hline Hole & Core & Section & $\begin{array}{l}\text { Sampled } \\
\text { at }(\mathrm{cm})\end{array}$ & $\begin{array}{l}\text { Sand } \\
(\%)\end{array}$ & $\begin{array}{l}\text { Silt } \\
(\%)\end{array}$ & $\begin{array}{l}\text { Clay } \\
(\%)\end{array}$ & Classification \\
\hline 14 & 6 & 1 & $2-4$ & 1.6 & 58.8 & 39.6 & Clayey silt \\
\hline 14 & 6 & 2 & $3-5$ & 2.6 & 53.5 & 43.9 & Clayey silt \\
\hline 14 & 6 & 3 & $2-4$ & 2.5 & 53.3 & 44.2 & Clayey silt \\
\hline 14 & 6 & 4 & $2-4$ & 2.4 & 54.3 & 43.3 & Clayey silt \\
\hline 14 & 6 & 5 & $2-4$ & 1.6 & 60.2 & 38.2 & Clayey silt \\
\hline 14 & 6 & 6 & $2-4$ & 1.7 & 54.1 & 44.2 & Clayey silt \\
\hline 14 & 7 & 1 & $2-4$ & 2.7 & 59.0 & 38.3 & Clayey silt \\
\hline 14 & 7 & 2 & $3-5$ & 2.1 & 56.5 & 41.4 & Clayey silt \\
\hline 14 & 7 & 3 & $2-4$ & 1.8 & 54.1 & 44.1 & Clayey silt \\
\hline 14 & 7 & 4 & $2-4$ & 2.1 & 52.7 & 45.2 & Clayey silt \\
\hline 14 & 7 & 5 & $2-4$ & 3.3 & 51.9 & 44.8 & Clayey silt \\
\hline 14 & 7 & 6 & $2-4$ & 0.9 & 54.7 & 44.4 & Clayey silt \\
\hline 14 & 8 & 1 & $2-4$ & 2.6 & 48.0 & 49.4 & Silty clay \\
\hline 14 & 8 & 2 & $3-5$ & 2.4 & 48.5 & 49.1 & Silty clay \\
\hline 14 & 8 & 3 & $2-4$ & 2.5 & 46.7 & 50.8 & Silty clay \\
\hline 14 & 8 & 4 & $148-150$ & 2.7 & 52.9 & 44.4 & Clayey silt \\
\hline 14 & 8 & 5 & $2-4$ & 2.4 & 50.1 & 47.5 & Clayey silt \\
\hline 14 & 8 & 6 & $148-150$ & 1.6 & 55.6 & 42.8 & Clayey silt \\
\hline 14 & 9 & 2 & $3-5$ & 1.3 & 47.0 & 51.7 & Silty clay \\
\hline 14 & 9 & 3 & $148-150$ & 0.9 & 54.7 & 44.4 & Clayey silt \\
\hline 14 & 9 & 4 & $20-22$ & 1.4 & 51.6 & 47.0 & Clayey silt \\
\hline 14 & 9 & 5 & $8-10$ & 0.5 & 57.3 & 42.2 & Clayey silt \\
\hline 14 & 9 & 6 & $8-10$ & 1.2 & 57.1 & 41.7 & Clayey silt \\
\hline 15 & 1 & 2 & $2-4$ & 6.6 & 11.5 & 81.9 & Clay \\
\hline 15 & 1 & 3 & $2-4$ & 12.3 & 16.2 & 71.5 & Silty clay \\
\hline 15 & 1 & 4 & $2-4$ & 22.7 & 20.6 & 56.7 & Sand silt clay \\
\hline 15 & 1 & 5 & $2-4$ & 13.1 & 22.0 & 64.9 & Silty clay \\
\hline 15 & 1 & 6 & $2-4$ & 12.5 & 20.8 & 66.7 & Silty clay \\
\hline 15 & 2 & 1 & $2-4$ & 14.9 & 19.9 & 65.2 & Silty clay \\
\hline 15 & 2 & 2 & $2-4$ & 12.5 & 20.0 & 67.5 & Silty clay \\
\hline 15 & 2 & 3 & $2-4$ & 12.1 & 14.4 & 73.5 & Silty clay \\
\hline 15 & 2 & 4 & $2-4$ & 14.4 & 19.4 & 66.2 & Silty clay \\
\hline 15 & 2 & 5 & $2-4$ & 13.4 & 19.0 & 67.6 & Silty clay \\
\hline 15 & 2 & 6 & $2-4$ & 9.8 & 18.7 & 71.5 & Silty clay \\
\hline 15 & 3 & 1 & $2-4$ & 6.5 & 35.5 & 58.0 & Silty clay \\
\hline 15 & 3 & 2 & $2-4$ & 4.0 & 36.1 & 59.9 & Silty clay \\
\hline 15 & 3 & 4 & $2-4$ & 4.2 & 36.7 & 59.1 & Silty clay \\
\hline
\end{tabular}




\begin{tabular}{|c|c|c|c|c|c|c|c|}
\hline Hole & Core & Section & $\begin{array}{l}\text { Sampled } \\
\text { at }(\mathrm{cm})\end{array}$ & $\begin{array}{l}\text { Sand } \\
(\%)\end{array}$ & $\begin{array}{l}\text { Silt } \\
(\%)\end{array}$ & $\begin{array}{l}\text { Clay } \\
(\%)\end{array}$ & Classification \\
\hline 15 & 3 & 5 & $2-4$ & 4.4 & 36.5 & 59.1 & Silty clay \\
\hline 15 & 3 & 6 & $2-4$ & 3.6 & 38.0 & 58.4 & Silty clay \\
\hline 15 & 4 & 1 & $2-4$ & 2.9 & 37.9 & 59.2 & Silty clay \\
\hline 15 & 4 & 3 & $2-4$ & 2.6 & 40.1 & 57.3 & Silty clay \\
\hline 15 & 4 & 4 & $2-4$ & 3.6 & 40.4 & 56.0 & Silty clay \\
\hline 15 & 4 & 5 & $2-4$ & 3.2 & 43.9 & 52.9 & Silty clay \\
\hline 15 & 4 & 6 & $2 \cdot 4$ & 2.4 & 38.7 & 58.9 & Silty clay \\
\hline 15 & 5 & 1 & $2-4$ & 2.0 & 40.5 & 57.5 & Silty clay \\
\hline 15 & 5 & 2 & $2-4$ & 2.3 & 29.0 & 68.7 & Silty clay \\
\hline 15 & 5 & 3 & $2-4$ & 3.7 & 30.7 & 65.6 & Silty clay \\
\hline 15 & 5 & 4 & $2-4$ & 4.4 & 46.6 & 49.0 & Silty clay \\
\hline 15 & 5 & 5 & $2-4$ & 4.6 & 35.4 & 60.0 & Silty clay \\
\hline 15 & 5 & 6 & $2-4$ & 2.9 & 45.9 & 51.2 & Silty clay \\
\hline 15 & 6 & 1 & $13-15$ & 0.4 & 30.8 & 68.8 & Silty clay \\
\hline 15 & 6 & 2 & $17-19$ & 0.5 & 32.1 & 67.4 & Silty clay \\
\hline 15 & 6 & 3 & $2-4$ & 0.5 & 28.1 & 71.4 & Silty clay \\
\hline 15 & 6 & 4 & $2-4$ & 2.2 & 31.3 & 66.5 & Silty clay \\
\hline 15 & 6 & 5 & $2-4$ & 0.8 & 51.4 & 47.8 & Clayey silt \\
\hline 15 & 6 & 6 & $2-4$ & 1.5 & 46.0 & 52.5 & Silty clay \\
\hline 15 & 7 & 1 & $2-4$ & 2.3 & 48.1 & 49.6 & Silty clay \\
\hline 15 & 7 & 2 & $4-6$ & 1.5 & 48.1 & 50.4 & Silty clay \\
\hline 15 & 7 & 3 & $2-4$ & 2.5 & 47.7 & 49.8 & Silty clay \\
\hline 15 & 7 & 4 & $2-4$ & 1.7 & 62.6 & 35.7 & Clayey silt \\
\hline 15 & 7 & 5 & $2-4$ & 2.4 & 54.0 & 43.6 & Clayey silt \\
\hline 15 & 7 & 6 & $2-4$ & 1.7 & 40.2 & 58.1 & Silty clay \\
\hline 15 & 8 & 1 & $148-150$ & 7.0 & 63.2 & 29.8 & Clayey silt \\
\hline 15 & 8 & 2 & $2-4$ & 9.3 & 68.3 & 22.4 & Clayey silt \\
\hline 15 & 8 & 3 & $2-4$ & 9.9 & 45.8 & 44.3 & Clayey silt \\
\hline 15 & 8 & 4 & $2-4$ & 7.9 & 43.5 & 48.6 & Silty clay \\
\hline 15 & 8 & 5 & $2-4$ & 10.4 & 44.2 & 45.4 & Silty clay \\
\hline 15 & 8 & 6 & $2-4$ & 13.4 & 60.0 & 26.6 & Clayey silt \\
\hline 15 & 9 & 1 & $48-50$ & 6.5 & 47.5 & 46.0 & Clayey silt \\
\hline 15 & 9 & 2 & $6-8$ & 12.1 & 58.5 & 29.4 & Clayey silt \\
\hline 15 & 9 & 3 & $2-4$ & 7.7 & 52.0 & 40.3 & Clayey silt \\
\hline 15 & 9 & 4 & $2-4$ & 7.8 & 62.5 & 29.7 & Clayey silt \\
\hline 15 & 9 & 5 & $2-4$ & 3.8 & 51.5 & 44.7 & Clayey silt \\
\hline 15 & 9 & 6 & $2-4$ & 7.6 & 60.1 & 32.3 & Clayey silt \\
\hline
\end{tabular}


TABLE 1 - Continued

\begin{tabular}{|c|c|c|c|c|c|c|c|}
\hline Hole & Core & Section & $\begin{array}{l}\text { Sampled } \\
\text { at }(\mathrm{cm})\end{array}$ & $\begin{array}{l}\text { Sand } \\
(\%)\end{array}$ & $\begin{array}{l}\text { Silt } \\
(\%)\end{array}$ & $\begin{array}{l}\text { Clay } \\
(\%)\end{array}$ & Classification \\
\hline 16 & 1 & 1 & $2-4$ & 42.0 & 29.1 & 28.9 & Sand silt clay \\
\hline 16 & 1 & 2 & $2-4$ & 48.4 & 29.3 & 22.3 & Sand silt clay \\
\hline 16 & 1 & 4 & $0-3$ & 24.1 & 19.5 & 56.4 & Sandy clay \\
\hline 16 & 1 & 6 & $2-4$ & 9.6 & 15.0 & 75.4 & Clay \\
\hline 16 & 2 & 1 & $148-150$ & 31.2 & 24.5 & 44.3 & Sand silt clay \\
\hline 16 & 2 & 2 & $148-150$ & 31.0 & 22.6 & 46.4 & Sand silt clay \\
\hline 16 & 2 & 3 & $148-150$ & 27.0 & 21.7 & 51.3 & Sand silt clay \\
\hline 16 & 2 & 4 & $148-150$ & 18.8 & 20.4 & 60.8 & Silty clay \\
\hline 16 & 2 & 5 & $148-150$ & 19.6 & 17.6 & 62.8 & Sandy clay \\
\hline 16 & 2 & 6 & $2-4$ & 14.7 & 15.1 & 70.2 & Silty clay \\
\hline 16 & 3 & 1 & $148-150$ & 9.4 & 31.1 & $59: 5$ & Silty clay \\
\hline 16 & 3 & 2 & $148-150$ & 13.5 & 30.5 & 56.0 & Silty clay \\
\hline 16 & 3 & 3 & $148-150$ & 15.0 & 25.0 & 60.0 & Silty clay \\
\hline 16 & 3 & 4 & $148-150$ & 10.9 & 24.2 & 64.9 & Silty clay \\
\hline 16 & 3 & 5 & $148-150$ & 13.4 & 26.0 & 60.6 & Silty clay \\
\hline 16 & 3 & 6 & $148-150$ & 13.1 & 22.2 & 64.7 & Silty clay \\
\hline 16 & 4 & 1 & $2-4$ & 6.0 & 33.4 & 60.6 & Silty clay \\
\hline 16 & 4 & 2 & $2-4$ & 7.0 & 32.7 & 60.3 & Silty clay \\
\hline 16 & 4 & 3 & $2-4$ & 4.6 & 31.1 & 64.3 & Silty clay \\
\hline 16 & 4 & 4 & $2-4$ & 8.0 & 35.4 & 56.6 & Silty clay \\
\hline 16 & 4 & 5 & $2-4$ & 8.9 & 43.9 & 47.2 & Silty clay \\
\hline 16 & 4 & 6 & $148-150$ & 6.9 & 32.3 & 60.8 & Silty clay \\
\hline 16 & 5 & 1 & $148-150$ & 2.6 & 35.5 & 61.9 & Silty clay \\
\hline 16 & 5 & 2 & $148-150$ & 2.7 & 36.6 & 60.7 & Silty clay \\
\hline 16 & 5 & 3 & $148-150$ & 2.7 & 36.0 & 61.3 & Silty clay \\
\hline 16 & 5 & 4 & $148-150$ & 3.5 & 31.5 & 65.0 & Silty clay \\
\hline 16 & 5 & 5 & $148-150$ & 4.5 & 32.3 & 63.2 & Silty clay \\
\hline 16 & 5 & 6 & $148-150$ & 3.9 & 32.2 & 63.9 & Silty clay \\
\hline 16 & 6 & 1 & $148-150$ & 1.7 & 32.7 & 65.6 & Silty clay \\
\hline 16 & 6 & 2 & $148-150$ & 4.5 & 33.0 & 62.5 & Silty clay \\
\hline 16 & 6 & 3 & $148-150$ & 4.7 & 36.8 & 58.5 & Silty clay \\
\hline 16 & 6 & 4 & $148-150$ & 4.9 & 43.3 & 51.8 & Silty clay \\
\hline 16 & 6 & 5 & $148-150$ & 4.4 & 44.2 & 51.4 & Silty clay \\
\hline 16 & 6 & 6 & $148-150$ & 4.8 & 40.6 & 54.6 & Silty clay \\
\hline 16 & 7 & 1 & $148-150$ & 1.6 & 33.6 & 64.8 & Silty clay \\
\hline 16 & 7 & 2 & $148-150$ & 2.4 & 35.0 & 62.6 & Silty clay \\
\hline 16 & 7 & 3 & $148-150$ & 0.8 & 33.8 & 65.4 & Silty clay \\
\hline
\end{tabular}


TABLE 1 - Continued

\begin{tabular}{|c|c|c|c|c|c|c|c|}
\hline Hole & Core & Section & $\begin{array}{l}\text { Sampled } \\
\text { at }(\mathrm{cm})\end{array}$ & $\begin{array}{l}\text { Sand } \\
(\%)\end{array}$ & $\begin{array}{l}\text { Silt } \\
(\%)\end{array}$ & $\begin{array}{l}\text { Clay } \\
(\%)\end{array}$ & Classification \\
\hline 16 & 7 & 4 & $148-150$ & 0.8 & 38.4 & 60.8 & Silty clay \\
\hline 16 & 7 & 5 & $148-150$ & 0.9 & 39.2 & 59.9 & Silty clay \\
\hline 16 & 7 & 6 & $148-150$ & 2.3 & 37.7 & 60.0 & Silty clay \\
\hline 16 & 8 & 1 & $148-150$ & 1.5 & 30.9 & 67.6 & Silty clay \\
\hline 16 & 8 & 2 & $148-150$ & 0.8 & 32.6 & 66.6 & Silty clay \\
\hline 16 & 8 & 3 & $148-150$ & 0.4 & 33.4 & 66.2 & Silty clay \\
\hline 16 & 8 & 4 & $148-150$ & 0.9 & 31.5 & 67.6 & Silty clay \\
\hline 16 & 8 & 5 & $148-150$ & 1.4 & 32.8 & 65.8 & Silty clay \\
\hline 16 & 8 & 6 & $148-150$ & 0.8 & 32.1 & 67.1 & Silty clay \\
\hline 16 & 9 & 1 & $148-150$ & 3.2 & 48.6 & 48.2 & Clayey silt \\
\hline 16 & 9 & 2 & $148-150$ & 5.1 & 46.7 & 48.2 & Silty clay \\
\hline 16 & 9 & 3 & $148-150$ & 4.8 & 42.3 & 52.9 & Silty clay \\
\hline 16 & 9 & 4 & $148-150$ & 3.6 & 46.0 & 50.4 & Silty clay \\
\hline 16 & 9 & 5 & $2-4$ & 2.4 & 41.4 & 56.2 & Silty clay \\
\hline 16 & 9 & 6 & $148-150$ & 2.9 & 42.4 & 54.7 & Silty clay \\
\hline 16 & 10 & 1 & $148-150$ & 3.8 & 44.4 & 51.8 & Silty clay \\
\hline 16 & 10 & 2 & $148-150$ & 5.5 & 49.7 & 44.8 & Clayey silt \\
\hline 16 & 10 & 4 & $148-150$ & 7.1 & 40.5 & 52.4 & Silty clay \\
\hline 16 & 10 & 5 & $148-150$ & 6.6 & 45.7 & 47.7 & Silty clay \\
\hline 16 & 10 & 6 & $148-150$ & 15.1 & 42.8 & 42.1 & Clayey silt \\
\hline 16 & 11 & 1 & $148-150$ & 12.4 & 43.2 & 44.4 & Silty clay \\
\hline 16 & 11 & 2 & $148-150$ & 13.1 & 36.9 & 50.0 & Silty clay \\
\hline 16 & 11 & 3 & $148-150$ & 15.1 & 33.5 & 51.4 & Silty clay \\
\hline 16 & 11 & 4 & $148-150$ & 6.8 & 46.2 & 47.0 & Silty clay \\
\hline 16 & 11 & 5 & $148-150$ & 11.4 & 43.5 & 45.1 & Silty clay \\
\hline 16 & 11 & 6 & $148-150$ & 5.9 & 51.2 & 42.9 & Clayey silt \\
\hline 17 & 1 & 1 & $5-7$ & 32.1 & 16.2 & 51.7 & Sandy clay \\
\hline 17 & 1 & 2 & $6-8$ & 19.7 & 20.7 & 59.6 & Silty clay \\
\hline 17 & 1 & 3 & $6-8$ & 33.0 & 19.8 & 47.2 & Sandy clay \\
\hline 17 & 1 & 4 & $8-10$ & 13.7 & 25.9 & 60.4 & Silty clay \\
\hline 17 & 1 & 5 & $8-10$ & 25.6 & 21.8 & 52.6 & Sand silt clay \\
\hline 17 & 2 & 1 & $25-27$ & 6.4 & 38.2 & 55.4 & Silty clay \\
\hline 17 & 2 & 2 & $8-10$ & 4.0 & 41.5 & 54.5 & Silty clay \\
\hline 17 & 2 & 3 & $8-10$ & 6.3 & 39.3 & 54.4 & Silty clay \\
\hline 17 & 2 & 4 & $11-13$ & 4.2 & 45.7 & 50.1 & Silty clay \\
\hline 17 & 2 & 5 & $13-15$ & 3.7 & 41.3 & 55.0 & Silty clay \\
\hline 17 & 2 & 6 & $8-10$ & 1.8 & 37.8 & 60.4 & Silty clay \\
\hline
\end{tabular}


TABLE 1 - Continued

\begin{tabular}{|c|c|c|c|c|c|c|c|}
\hline Hole & Core & Section & $\begin{array}{l}\text { Sampled } \\
\text { at }(\mathrm{cm})\end{array}$ & $\begin{array}{l}\text { Sand } \\
(\%)\end{array}$ & $\begin{array}{l}\text { Silt } \\
(\%)\end{array}$ & $\begin{array}{l}\text { Clay } \\
(\%)\end{array}$ & Classification \\
\hline $17 \mathrm{~A}$ & 1 & 1 & $6-8$ & 0.9 & 50.6 & 48.5 & Clayey silt \\
\hline $17 \mathrm{~A}$ & 1 & 2 & $8-10$ & 4.0 & 50.0 & 46.0 & Clayey silt \\
\hline $17 \mathrm{~A}$ & 1 & 3 & $20-22$ & 3.4 & 52.9 & 43.7 & Clayey silt \\
\hline $17 \mathrm{~A}$ & 1 & 4 & $10-12$ & 1.1 & 49.5 & 49.4 & Clayey silt \\
\hline $17 \mathrm{~A}$ & 1 & 5 & $10-12$ & 0.4 & 40.7 & 58.9 & Silty clay \\
\hline $17 \mathrm{~A}$ & 1 & 6 & $12-14$ & 0.6 & 50.2 & 49.2 & Clayey silt \\
\hline $17 \mathrm{~A}$ & 2 & 1 & $11-13$ & 0.2 & 47.5 & 52.3 & Silty clay \\
\hline $17 \mathrm{~A}$ & 2 & 2 & $18-20$ & 0.8 & 48.4 & 50.8 & Silty clay \\
\hline $17 \mathrm{~A}$ & 2 & 3 & $18-20$ & 0.3 & 53.9 & 45.8 & Clayey silt \\
\hline $17 \mathrm{~A}$ & 2 & 4 & $18-20$ & 0.1 & 54.8 & 45.1 & Clayey silt \\
\hline $17 \mathrm{~A}$ & 2 & 5 & $11-13$ & 4.4 & 42.3 & 53.3 & Silty clay \\
\hline $17 \mathrm{~A}$ & 2 & 6 & $15-17$ & 6.1 & 45.5 & 48.4 & Silty clay \\
\hline $17 \mathrm{~A}$ & 3 & 1 & $80-82$ & 1.3 & 72.9 & 25.8 & Clayey silt \\
\hline $17 \mathrm{~A}$ & 3 & 6 & $148-150$ & 3.8 & 38.6 & 57.6 & Silty clay \\
\hline $17 \mathrm{~A}$ & 4 & 5 & $0-2$ & 1.7 & 53.5 & 44.8 & Clayey silt \\
\hline $17 \mathrm{~A}$ & 4 & 6 & $75-77$ & 5.4 & 47.5 & 47.1 & Clayey silt \\
\hline $17 \mathrm{~B}$ & 1 & 1 & $10-12$ & 2.6 & 47.6 & 49.8 & Silty clay \\
\hline 17B & 1 & 2 & $11-13$ & 0.9 & 49.4 & 49.7 & Silty clay \\
\hline 17B & 1 & 3 & $11-13$ & 0.8 & 47.2 & 52.0 & Silty clay \\
\hline 17B & 1 & 4 & $11-13$ & 3.6 & 48.2 & 48.2 & Silt and clay \\
\hline 17B & 1 & 5 & $78-80$ & 2.3 & 53.9 & 43.8 & Clayey silt \\
\hline 17B & 1 & 6 & $78-80$ & 2.9 & 52.5 & 44.6 & Clayey silt \\
\hline 17B & 2 & 1 & $24-26$ & 3.0 & 57.2 & 39.8 & Clayey silt \\
\hline 17B & 2 & 2 & $10-12$ & 3.9 & 57.7 & 38.4 & Clayey silt \\
\hline 17B & 2 & 3 & $15-17$ & 3.5 & 65.4 & 31.1 & Clayey silt \\
\hline 17B & 2 & 4 & $11-13$ & 3.2 & 57.4 & 39.4 & Clayey silt \\
\hline $17 \mathrm{~B}$ & 2 & 5 & $10-12$ & 4.1 & 55.8 & 40.1 & Clayey silt \\
\hline 17B & 2 & 6 & $12-14$ & 4.7 & 55.7 & 39.6 & Clayey silt \\
\hline 17B & 3 & 1 & $10-12$ & 3.9 & 51.0 & 45.1 & Clayey silt \\
\hline $17 \mathrm{~B}$ & 3 & 2 & $10-12$ & 4.8 & 48.1 & 47.1 & Clayey silt \\
\hline 17B & 3 & 3 & $10-12$ & 4.6 & 48.1 & 47.3 & Clayey silt \\
\hline 17B & 3 & 4 & $10-12$ & 4.9 & 49.2 & 45.9 & Clayey silt \\
\hline $17 \mathrm{~B}$ & 3 & 5 & $10-12$ & 4.1 & 50.4 & 45.5 & Clayey silt \\
\hline 17B & 3 & 6 & $78-80$ & 4.1 & 52.0 & 43.9 & Clayey silt \\
\hline 17B & 4 & 1 & $80-82$ & 1.9 & 56.1 & 42.0 & Clayey silt \\
\hline $17 \mathrm{~B}$ & 4 & 3 & $13-15$ & 2.5 & 51.9 & 45.6 & Clayey silt \\
\hline
\end{tabular}


TABLE 1 - Continued

\begin{tabular}{|c|c|c|c|c|c|c|c|}
\hline Hole & Core & Section & $\begin{array}{l}\text { Sampled } \\
\text { at }(\mathrm{cm})\end{array}$ & $\begin{array}{l}\text { Sand } \\
(\%)\end{array}$ & $\begin{array}{l}\text { Silt } \\
(\%)\end{array}$ & $\begin{array}{l}\text { Clay } \\
(\%)\end{array}$ & Classification \\
\hline 17B & 4 & 4 & $13-15$ & 1.9 & 55.6 & 42.5 & Clayey silt \\
\hline $17 \mathrm{~B}$ & 4 & 5 & $13-15$ & 2.4 & 53.9 & 43.7 & Clayey silt \\
\hline $17 \mathrm{~B}$ & 4 & 6 & $18-20$ & 0.7 & 44.2 & 55.1 & Silty clay \\
\hline 18 & 1 & 5 & $25-27$ & 7.9 & 12.5 & 79.6 & Clay \\
\hline 18 & 2 & 1 & $0-2$ & 2.0 & 59.2 & 38.8 & Clayey silt \\
\hline 18 & 2 & 2 & $13-15$ & 0.5 & 65.0 & 34.5 & Clayey silt \\
\hline 18 & 2 & 3 & $148-150$ & 3.6 & 56.2 & 40.2 & Clayey silt \\
\hline 18 & 2 & 4 & $148-150$ & 1.4 & 60.8 & 37.8 & Clayey silt \\
\hline 18 & 2 & 5 & $148-150$ & 3.6 & 53.9 & 42.5 & Clayey silt \\
\hline 18 & 2 & 6 & $148-150$ & 3.4 & 52.0 & 44.6 & Clayey silt \\
\hline 18 & 3 & 1 & $148-150$ & 2.8 & 51.7 & 45.5 & Clayey silt \\
\hline 18 & 3 & 2 & $13-15$ & 3.9 & 54.0 & 42.1 & Clayey silt \\
\hline 18 & 3 & 3 & $148-150$ & 5.4 & 54.1 & 40.5 & Clayey silt \\
\hline 18 & 3 & 4 & $148-150$ & 3.6 & 58.0 & 38.4 & Clayey silt \\
\hline 18 & 3 & 5 & $148-150$ & 4.6 & 58.1 & 37.3 & Clayey silt \\
\hline 18 & 3 & 6 & $148-150$ & 3.9 & 56.7 & 39.4 & Clayey silt \\
\hline 18 & 4 & 2 & $0-2$ & 5.1 & 53.4 & 41.5 & Clayey silt \\
\hline 18 & 4 & 3 & $0-2$ & 5.3 & 52.7 & 42.0 & Clayey silt \\
\hline 18 & 4 & 4 & 13-15 & 12.2 & 38.5 & 49.3 & Silty clay \\
\hline 18 & 4 & 5 & $148-150$ & 7.0 & 47.2 & 45.8 & Clayey silt \\
\hline 18 & 5 & 1 & $148-150$ & 12.3 & 42.0 & 45.7 & Silty clay \\
\hline 18 & 5 & 3 & $148-150$ & 12.8 & 35.3 & 51.9 & Silty clay \\
\hline 18 & 5 & 5 & $0-2$ & 12.4 & 44.3 & 43.3 & Clayey silt \\
\hline 18 & 5 & 6 & $0-2$ & 11.0 & 44.7 & 44.3 & Clayey silt \\
\hline 18 & 6 & 1 & $13-15$ & 14.9 & 44.4 & 40.7 & Clayey silt \\
\hline 18 & 6 & 2 & $148-150$ & 10.8 & 47.5 & 41.7 & Clayey silt \\
\hline 18 & 6 & 3 & $148-150$ & 9.1 & 49.6 & 41.3 & Clayey silt \\
\hline 18 & 6 & 4 & $148=150$ & 11.9 & 46.3 & 41.8 & Clayey silt \\
\hline 18 & 6 & 6 & $75-77$ & 13.9 & 52.6 & 33.5 & Clayey silt \\
\hline 19 & 1 & 1 & $78-80$ & 3.8 & 19.0 & 77.2 & Clay \\
\hline 19 & 1 & 2 & $2-4$ & 0.5 & 16.9 & 82.6 & Clay \\
\hline 19 & 1 & 3 & $1-3$ & 0.3 & 20.0 & 79.7 & Clay \\
\hline 19 & 1 & 4 & $1-3$ & 0.2 & 18.1 & 81.7 & Clay \\
\hline 19 & 1 & 5 & $1-3$ & 0.5 & 19.7 & 79.8 & Clay \\
\hline 19 & 1 & 6 & $1-3$ & 0.5 & 20.9 & 78.6 & Clay \\
\hline 19 & 2 & 1 & $15-17$ & 0.2 & 24.1 & 75.7 & Clay \\
\hline
\end{tabular}


TABLE 1 - Continued

\begin{tabular}{|c|c|c|c|c|c|c|c|}
\hline Hole & Core & Section & $\begin{array}{l}\text { Sampled } \\
\text { at }(\mathrm{cm})\end{array}$ & $\begin{array}{l}\text { Sand } \\
(\%)\end{array}$ & $\begin{array}{l}\text { Silt } \\
(\%)\end{array}$ & $\begin{array}{l}\text { Clay } \\
(\%)\end{array}$ & Classification \\
\hline 19 & 2 & 2 & $1-3$ & 0.1 & 24.4 & 75.5 & Clay \\
\hline 19 & 2 & 3 & $148-150$ & 0.2 & 24.2 & 75.6 & Clay \\
\hline 19 & 2 & 4 & $148-150$ & 0.1 & 26.9 & 73.0 & Silty clay \\
\hline 19 & 2 & 5 & $148-150$ & 0.1 & 24.6 & 75.3 & Clay \\
\hline 19 & 2 & 6 & $148-150$ & 0.2 & 23.5 & 76.3 & Clay \\
\hline 19 & 3 & 1 & $33-35$ & 0.2 & 43.8 & 56.0 & Silty clay \\
\hline 19 & 3 & 2 & $1-3$ & 0.2 & 39.4 & 60.4 & Silty clay \\
\hline 19 & 3 & 3 & $1-3$ & 0.2 & 41.1 & 58.7 & Silty clay \\
\hline 19 & 3 & 4 & $1-3$ & 0.1 & 36.4 & 63.5 & Silty clay \\
\hline 19 & 3 & 5 & $1-3$ & 0.2 & 46.4 & 53.4 & Silty clay \\
\hline 19 & 3 & 6 & $1-3$ & 0.1 & 27.7 & 72.2 & Silty clay \\
\hline 19 & 4 & 1 & $148-150$ & 5.8 & 39.3 & 54.9 & Silty clay \\
\hline 19 & 4 & 2 & $1-3$ & 0.2 & 44.7 & 55.1 & Silty clay \\
\hline 19 & 4 & 3 & $148-150$ & 0.2 & 49.2 & 50.6 & Silty clay \\
\hline 19 & 4 & 4 & $148-150$ & 1.2 & 47.9 & 50.9 & Silty clay \\
\hline 19 & 4 & 5 & $148-150$ & 0.4 & 51.3 & 48.3 & Clayey silt \\
\hline 19 & 4 & 6 & $148-150$ & 0.7 & 45.2 & 54.1 & Silty clay \\
\hline 19 & 5 & 1 & $148-150$ & 0.2 & 54.1 & 45.7 & Clayey silt \\
\hline 19 & 5 & 2 & $1-3$ & 0.2 & 53.6 & 46.2 & Clayey silt \\
\hline 19 & 5 & 3 & $3-5$ & 0.2 & 46.7 & 53.1 & Silty clay \\
\hline 19 & 5 & 4 & $25-27$ & 0.2 & 53.7 & 46.1 & Clayey silt \\
\hline 19 & 5 & 5 & $3-5$ & 0.2 & 57.1 & 42.7 & Clayey silt \\
\hline 19 & 5 & 6 & $3-5$ & 0.1 & 52.4 & 47.5 & Clayey silt \\
\hline 19 & 6 & 1 & $148-150$ & 0.6 & 40.3 & 59.1 & Silty clay \\
\hline 19 & 6 & 2 & $148-150$ & 0.8 & 42.3 & 56.9 & Silty clay \\
\hline 19 & 6 & 3 & $148-150$ & 0.1 & 46.0 & 53.9 & Silty clay \\
\hline 19 & 6 & 4 & $148-150$ & 0.9 & 42.3 & 56.8 & Silty clay \\
\hline 19 & 6 & 5 & $148-150$ & 0.2 & 54.7 & 45.1 & Clayey silt \\
\hline 19 & 6 & 6 & $148-150$ & 0.9 & 54.5 & 44.6 & Clayey silt \\
\hline 19 & 7 & 1 & $2-4$ & 0.6 & 46.5 & 52.9 & Silty clay \\
\hline 19 & 7 & 3 & $2-4$ & 0.3 & 53.4 & 46.3 & Clayey silt \\
\hline 19 & 7 & 4 & $2-4$ & 0.4 & 36.8 & 62.8 & Silty clay \\
\hline 19 & 7 & 5 & $3-5$ & 0.7 & 48.3 & 51.0 & Silty clay \\
\hline 19 & 7 & 6 & $3-5$ & 0.9 & 49.9 & 49.2 & Clayey silt \\
\hline 19 & 8 & 1 & $148-150$ & 0.5 & 55.6 & 43.9 & Clayey silt \\
\hline 19 & 8 & 3 & $148-150$ & 0.5 & 50.7 & 48.8 & Clayey silt \\
\hline
\end{tabular}


TABLE 1 - Continued

\begin{tabular}{|c|c|c|c|c|c|c|c|}
\hline Hole & Core & Section & $\begin{array}{l}\text { Sampled } \\
\text { at }(\mathrm{cm})\end{array}$ & $\begin{array}{l}\text { Sand } \\
(\%)\end{array}$ & $\begin{array}{l}\text { Silt } \\
(\%)\end{array}$ & $\begin{array}{l}\text { Clay } \\
(\%)\end{array}$ & Classification \\
\hline 19 & 8 & 4 & $148-150$ & 0.8 & 48.6 & 50.6 & Silty clay \\
\hline 19 & 8 & 5 & $148-150$ & 0.3 & 47.5 & 52.2 & Silty clay \\
\hline 19 & 8 & 6 & $148-150$ & 1.3 & 49.5 & 49.2 & Clayey silt \\
\hline 19 & 9 & 1 & $148-150$ & 0.5 & 46.3 & 53.2 & Silty clay \\
\hline 19 & 9 & 2 & $148-150$ & 1.8 & 46.0 & 52.2 & Silty clay \\
\hline 19 & 9 & 3 & $148-150$ & 1.7 & 47.9 & 50.4 & Silty clay \\
\hline 19 & 9 & 4 & $148-150$ & 2.4 & 52.3 & 45.3 & Clayey silt \\
\hline 19 & 9 & 5 & $148-150$ & 2.0 & 46.2 & 51.8 & Silty clay \\
\hline 19 & 9 & 6 & $6-8$ & 1.8 & 44.8 & 53.4 & Silty clay \\
\hline 19 & 10 & 1 & $148-150$ & 2.5 & 43.6 & 53.9 & Silty clay \\
\hline 19 & 10 & 2 & $148-150$ & 2.3 & 41.3 & 56.4 & Silty clay \\
\hline 19 & 10 & 3 & $148-150$ & 1.7 & 43.7 & 54.6 & Silty clay \\
\hline 19 & 10 & 5 & $148-150$ & 2.5 & 40.2 & 57.3 & Silty clay \\
\hline 19 & 10 & 6 & $148-150$ & 1.2 & 44.2 & 54.6 & Silty clay \\
\hline 19 & 11 & 1 & $148-150$ & 1.9 & 42.5 & 55.6 & Silty clay \\
\hline 19 & 11 & 2 & $148-150$ & 1.1 & 40.3 & 58.6 & Silty clay \\
\hline 19 & 11 & 3 & $148-150$ & 5.2 & 41.6 & 53.2 & Silty clay \\
\hline 19 & 11 & 4 & $148-150$ & 1.1 & 39.2 & 59.7 & Silty clay \\
\hline $20 \mathrm{~A}$ & 2 & 1 & $60-62$ & 0.3 & 35.6 & 64.1 & Silty clay \\
\hline $20 \mathrm{~A}$ & 2 & 2 & $1-3$ & 0.8 & 43.5 & 55.7 & Silty clay \\
\hline $20 \mathrm{~A}$ & 2 & 3 & $5-7$ & 0.4 & 33.1 & 66.5 & Silty clay \\
\hline $20 \mathrm{~A}$ & 2 & 4 & $148-150$ & 0.4 & 45.2 & 54.4 & Silty clay \\
\hline $20 \mathrm{~A}$ & 2 & 5 & $148-150$ & 0.3 & 38.9 & 60.8 & Silty clay \\
\hline $20 \mathrm{~A}$ & 2 & 6 & $148-150$ & 0.3 & 41.4 & 58.3 & Silty clay \\
\hline $20 \mathrm{~B}$ & 1 & 1 & $3-5$ & 0.2 & 34.5 & 65.3 & Silty clay \\
\hline $20 \mathrm{~B}$ & 1 & 2 & $7-9$ & 0.6 & 33.4 & 66.0 & Silty clay \\
\hline $20 \mathrm{~B}$ & 1 & 3 & $4-6$ & 0.3 & 33.6 & 66.1 & Silty clay \\
\hline $20 \mathrm{~B}$ & 1 & 4 & $3-5$ & 0.3 & 36.5 & 63.2 & Silty clay \\
\hline $20 \mathrm{~B}$ & 1 & 5 & $0-2$ & 0.3 & 31.4 & 68.3 & Silty clay \\
\hline $20 B$ & 1 & 6 & $1-3$ & 0.2 & 25.2 & 74.6 & Silty clay \\
\hline $20 \mathrm{C}$ & 1 & 1 & $1-3$ & 13.5 & 16.9 & 69.6 & Silty clay \\
\hline $20 \mathrm{C}$ & 1 & 2 & $1-3$ & 7.1 & 30.2 & 62.7 & Silty clay \\
\hline $20 \mathrm{C}$ & 1 & 3 & $1-3$ & 0.5 & 41.5 & 5.8 .0 & Silty clay \\
\hline $20 \mathrm{C}$ & 1 & 4 & $1-3$ & 1.7 & 38.1 & 60.2 & Silty clay \\
\hline $20 \mathrm{C}$ & 1 & 5 & $1-3$ & 0.4 & 39.4 & 60.2 & Silty clay \\
\hline $20 \mathrm{C}$ & 1 & 6 & $1-3$ & 0.7 & 37.8 & 61.5 & Silty clay \\
\hline
\end{tabular}


TABLE 1 - Continued

\begin{tabular}{|c|c|c|c|c|c|c|c|}
\hline Hole & Core & Section & $\begin{array}{l}\text { Sampled } \\
\text { at }(\mathrm{cm})\end{array}$ & $\begin{array}{l}\text { Sand } \\
(\%)\end{array}$ & $\begin{array}{l}\text { Silt } \\
(\%)\end{array}$ & $\begin{array}{l}\text { Clay } \\
(\%)\end{array}$ & Classification \\
\hline $20 \mathrm{C}$ & 2 & 1 & $148-150$ & 0.9 & 46.7 & 52.4 & Silty clay \\
\hline $20 \mathrm{C}$ & 2 & 2 & $148-150$ & 0.4 & 44.9 & 54.7 & Silty clay \\
\hline $20 \mathrm{C}$ & 2 & 3 & $20-22$ & 0.2 & 47.9 & 51.9 & Silty clay \\
\hline $20 \mathrm{C}$ & 2 & 4 & $6-8$ & 0.3 & 47.5 & 52.2 & Silty clay \\
\hline $20 \mathrm{C}$ & 2 & 5 & $148-150$ & 0.3 & 52.5 & 47.2 & Clayey silt \\
\hline $20 \mathrm{C}$ & 2 & 6 & $148-150$ & 0.5 & 46.8 & 52.7 & Silty clay \\
\hline $20 \mathrm{C}$ & 3 & 1 & $1-3$ & 0.4 & 37.3 & 62.3 & Silty clay \\
\hline $20 \mathrm{C}$ & 3 & 2 & $1-3$ & 0.4 & 16.0 & 83.6 & Clay \\
\hline $20 \mathrm{C}$ & 3 & 3 & $1-3$ & 0.5 & 25.1 & 74.4 & Silty clay \\
\hline $20 \mathrm{C}$ & 3 & 4 & $14-16$ & 0.5 & 22.4 & 77.1 & Clay \\
\hline $20 \mathrm{C}$ & 3 & 5 & $1-3$ & 0.5 & 21.3 & 78.2 & Clay \\
\hline $20 \mathrm{C}$ & 3 & 6 & $1-3$ & 0.5 & 23.0 & 76.5 & Clay \\
\hline $20 \mathrm{C}$ & 4 & 1 & $3-5$ & 0.5 & 27.0 & 72.5 & Silty clay \\
\hline $20 \mathrm{C}$ & 4 & 2 & $1-3$ & 0.2 & 26.3 & 73.5 & Silty clay \\
\hline $20 \mathrm{C}$ & 4 & 3 & $1-3$ & 0.2 & 28.0 & 71.8 & Silty clay \\
\hline $20 \mathrm{C}$ & 4 & 4 & $148-150$ & 0.4 & 26.2 & 73.4 & Silty clay \\
\hline $20 \mathrm{C}$ & 5 & 1 & $148-150$ & 0.6 & 43.3 & 56.1 & Silty clay \\
\hline $20 \mathrm{C}$ & 5 & 2 & $1-3$ & 0.1 & 91.6 & 8.3 & Silt \\
\hline $20 \mathrm{C}$ & 5 & 3 & $1-3$ & 0.3 & 43.1 & 56.6 & Silty clay \\
\hline $20 \mathrm{C}$ & 5 & 4 & $1-3$ & 0.8 & 45.7 & 53.5 & Silty clay \\
\hline $20 \mathrm{C}$ & 5 & 5 & $1-3$ & 5.3 & 47.6 & 47.1 & Clayey silt \\
\hline $20 \mathrm{C}$ & 5 & 6 & $1-3$ & 5.0 & 49.9 & 45.1 & Clayey silt \\
\hline $20 \mathrm{C}$ & 6 & 1 & $148-150$ & 0.8 & 40.3 & 58.0 & Silty clay \\
\hline $20 \mathrm{C}$ & 6 & 2 & $1-3$ & 1.0 & 43.3 & 55.7 & Silty clay \\
\hline $20 \mathrm{C}$ & 6 & 3 & $1-3$ & 1.4 & 46.9 & 51.7 & Silty clay \\
\hline $20 \mathrm{C}$ & 6 & 4 & $2-4$ & 3.0 & 44.8 & 52.2 & Silty clay \\
\hline $20 \mathrm{C}$ & 6 & 5 & $78-80$ & 4.8 & 31.3 & 63.0 & Silty clay \\
\hline 21 & 1 & 1 & $148-150$ & 40.9 & 25.0 & 34.1 & Sand silt clay \\
\hline 21 & 1 & 2 & $148-150$ & 49.9 & 20.9 & 29.2 & Sand silt clay \\
\hline 21 & 1 & 3 & $148-150$ & 39.2 & 16.6 & 44.2 & Sandy clay \\
\hline 21 & 1 & 4 & $11-13$ & 59.9 & 20.4 & 19.7 & Silty sand \\
\hline 21 & 1 & 5 & $148-150$ & 48.3 & 14.4 & 37.3 & Clayey sand \\
\hline 21 & 1 & 6 & $148-150$ & 56.3 & 17.7 & 26.0 & Clayey sand \\
\hline 21 & 2 & 1 & $148-150$ & 14.3 & 36.5 & 49.2 & Silty clay \\
\hline 21 & 2 & 2 & 0-2 & 15.0 & 43.3 & 41.7 & Clayey silt \\
\hline 21 & 2 & 3 & $148-150$ & 14.0 & 37.5 & 48.5 & Silty clay \\
\hline
\end{tabular}


TABLE 1 - Continued

\begin{tabular}{|c|c|c|c|c|c|c|c|}
\hline Hole & Core & Section & $\begin{array}{l}\text { Sampled } \\
\text { at }(\mathrm{cm})\end{array}$ & $\begin{array}{l}\text { Sand } \\
(\%)\end{array}$ & $\begin{array}{l}\text { Silt } \\
(\%)\end{array}$ & $\begin{array}{l}\text { Clay } \\
(\%)\end{array}$ & Classification \\
\hline 21 & 2 & 4 & $39-41$ & 12.7 & 47.2 & 40.1 & Clayey silt \\
\hline 21 & 2 & 5 & $51-53$ & 12.0 & 46.2 & 41.8 & Clayey silt \\
\hline 21 & 2 & 6 & $16-18$ & 11.2 & 40.2 & 48.6 & Silty clay \\
\hline 21 & 3 & 1 & $148-150$ & 8.4 & 29.1 & 62.5 & Silty clay \\
\hline 21 & 3 & 2 & $148-150$ & 15.0 & 26.0 & 59.0 & Silty clay \\
\hline 21 & 3 & 3 & $148-150$ & 12.0 & 25.4 & 62.6 & Silty clay \\
\hline 21 & 3 & 4 & $148-150$ & 16.3 & 25.5 & 58.2 & Silty clay \\
\hline 21 & 3 & 5 & $14-16$ & 19.8 & 24.8 & 55.4 & Silty clay \\
\hline 21 & 3 & 6 & $148-150$ & 4.6 & 31.1 & 64.3 & Silty clay \\
\hline 21 & 4 & 1 & $148-150$ & 25.3 & 33.7 & 41.0 & Sand silt clay \\
\hline 21 & 4 & 2 & 0-2 & 17.4 & 33.1 & 49.5 & Silty clay \\
\hline 21 & 4 & 3 & $148-150$ & 17.8 & 32.5 & 49.7 & Silty clay \\
\hline 21 & 4 & 5 & $148-150$ & 17.2 & 33.7 & 49.1 & Silty clay \\
\hline 21 & 4 & 6 & $148-150$ & 14.7 & 31.2 & 54.1 & Silty clay \\
\hline 21 & 5 & 1 & $31-33$ & 9.1 & 32.6 & 58.3 & Silty clay \\
\hline 21 & 5 & 2 & $148-150$ & 10.0 & 31.2 & 58.8 & Silty clay \\
\hline 21 & 5 & 3 & $148-150$ & 18.1 & 28.9 & 53.0 & Silty clay \\
\hline 21 & 5 & 4 & $148-150$ & 12.7 & 30.8 & 56.5 & Silty clay \\
\hline 21 & 5 & 5 & 96-98 & 14.2 & 32.2 & 53.6 & Silty clay \\
\hline 21 & 5 & 6 & $148-150$ & 12.4 & 31.3 & 56.3 & Silty clay \\
\hline 21 & 6 & 3 & $7-9$ & 10.7 & 32.4 & 56.9 & Silty clay \\
\hline 21 & 6 & 4 & $2-4$ & 15.4 & 37.7 & 46.9 & Silty clay \\
\hline 21 & 6 & 5 & $3-5$ & 9.5 & 35.8 & 54.7 & Silty clay \\
\hline 21 & 6 & 6 & $3-5$ & 36.7 & 33.6 & 29.7 & Sand silt clay \\
\hline 21 & 7 & 1 & $148-150$ & 15.9 & 30.5 & 53.6 & Silty clay \\
\hline 21 & 7 & 2 & $148-150$ & 19.5 & 35.8 & 44.7 & Silty clay \\
\hline 21 & 7 & 3 & $148-150$ & 19.0 & 33.1 & 47.9 & Silty clay \\
\hline 21 & 7 & 4 & $148-150$ & 22.7 & 32.3 & 45.0 & Sand silt clay \\
\hline 21 & 7 & 5 & $148-150$ & 14.2 & 34.5 & 51.3 & Silty clay \\
\hline 21 & 7 & 6 & $148-150$ & 11.3 & 33.7 & 55.0 & Silty clay \\
\hline 21 & 8 & 1 & $0-2$ & 22.9 & 32.4 & 44.7 & Sand silt clay \\
\hline 21 & 8 & 2 & $148-150$ & 26.9 & 31.5 & 41.6 & Sand silt clay \\
\hline 21 & 8 & 3 & $148-150$ & 30.0 & 30.4 & 39.6 & Sand silt clay \\
\hline 21 & 8 & 4 & $148-150$ & 33.4 & 29.9 & 36.7 & Sand silt clay \\
\hline 21 & 8 & 5 & $148-150$ & 34.8 & 33.7 & 31.5 & Sand silt clay \\
\hline 21 & 8 & 6 & $34-36$ & 25.7 & 34.0 & 40.3 & Sand silt clay \\
\hline $21 \mathrm{~A}$ & 1 & 1 & $148-150$ & 15.4 & 45.4 & 39.2 & Clayey silt \\
\hline
\end{tabular}


TABLE 1. (Continued)

\begin{tabular}{|c|c|c|c|c|c|c|c|}
\hline Hole & Core & Section & $\begin{array}{l}\text { Sampled } \\
\text { at }(\mathrm{cm})\end{array}$ & $\begin{array}{l}\text { Sand } \\
(\%)\end{array}$ & $\begin{array}{l}\text { Silt } \\
(\%)\end{array}$ & $\begin{array}{l}\text { Clay } \\
(\%)\end{array}$ & Classification \\
\hline $21 \mathrm{~A}$ & 1 & 2 & $148-150$ & 19.8 & 45.3 & 34.9 & Clayey silt \\
\hline $21 \mathrm{~A}$ & 1 & 3 & $148-150$ & 16.8 & 45.0 & 38.2 & Clayey silt \\
\hline $21 \mathrm{~A}$ & 1 & 4 & $148-150$ & 26.1 & 41.1 & 32.8 & Sand silt clay \\
\hline $21 \mathrm{~A}$ & 1 & 5 & $148-150$ & 20.8 & 45.5 & 33.7 & Sand silt clay \\
\hline $21 \mathrm{~A}$ & 1 & 6 & $148-150$ & 9.0 & 54.6 & 36.4 & Clayey silt \\
\hline $21 \mathrm{~A}$ & 2 & 1 & $148-150$ & 9.3 & 58.1 & 32.6 & Clayey silt \\
\hline $21 \mathrm{~A}$ & 2 & 2 & $148-150$ & 8.6 & 60.1 & 31.3 & Clayey silt \\
\hline $21 \mathrm{~A}$ & 2 & 3 & $148-150$ & 10.2 & 57.7 & 32.1 & Clayey silt \\
\hline $21 \mathrm{~A}$ & 2 & 5 & $148-150$ & 17.3 & 51.8 & 30.9 & Clayey silt \\
\hline $21 \mathrm{~A}$ & 2 & 6 & $148-150$ & 15.6 & 53.4 & 31.0 & Clayey silt \\
\hline $21 \mathrm{~A}$ & 3 & 1 & $148-150$ & 21.9 & 39.1 & 39.0 & Sand silt clay \\
\hline $21 \mathrm{~A}$ & 3 & 2 & $148-150$ & 22.1 & 39.2 & 38.7 & Sand silt clay \\
\hline $21 \mathrm{~A}$ & 3 & 3 & $148-150$ & 23.8 & 39.0 & 37.2 & Sand silt clay \\
\hline $21 \mathrm{~A}$ & 3 & 4 & $148-150$ & 21.0 & 37.9 & 41.1 & Sand silt clay \\
\hline $21 \mathrm{~A}$ & 3 & 5 & $11-13$ & 16.3 & 40.0 & 43.7 & Silty clay \\
\hline $21 \mathrm{~A}$ & 3 & 6 & $40-42$ & 18.7 & 38.0 & 43.3 & Silty clay \\
\hline 22 & 1 & 1 & $148-150$ & 40.2 & 20.6 & 39.2 & Sand silt clay \\
\hline 22 & 1 & 2 & $148-150$ & 39.5 & 20.9 & 39.6 & Sand silt clay \\
\hline 22 & 1 & 3 & $148-150$ & 34.0 & 19.0 & 47.0 & Sandy clay \\
\hline 22 & 1 & 4 & $148-150$ & 42.1 & 16.5 & 41.4 & Clayey sand \\
\hline 22 & 1 & 6 & $33-35$ & 42.8 & 15.5 & 41.7 & Clayey sand \\
\hline 22 & 2 & 1 & $13-15$ & 15.0 & 34.7 & 50.3 & Silty clay \\
\hline 22 & 2 & 2 & $15-17$ & 16.8 & 39.0 & 44.2 & Silty clay \\
\hline 22 & 2 & 3 & $18-22$ & 23.2 & 37.0 & 39.8 & Sand silt clay \\
\hline 22 & 2 & 6 & 13.15 & 25.6 & 32.4 & 42.0 & Sand silt clay \\
\hline 22 & 3 & 1 & $148-150$ & 12.0 & 42.0 & 46.0 & Silty clay \\
\hline 22 & 3 & 3 & $148-150$ & 9.7 & 44.2 & 46.1 & Silty clay \\
\hline 22 & 3 & 4 & $148-150$ & 10.2 & 45.1 & 44.7 & Clayey silt \\
\hline 22 & 3 & 5 & $148-150$ & 10.4 & 44.8 & 44.8 & Silt and clay \\
\hline 22 & 3 & 6 & $43-45$ & 7.6 & 44.0 & 48.4 & Silty clay \\
\hline 22 & 4 & 1 & $121-123$ & 0.2 & 75.2 & 24.6 & Silt \\
\hline 22 & 4 & 2 & $45-47$ & 0.1 & 71.0 & 28.0 & Clayey silt \\
\hline 22 & 4 & 2 & $10-12$ & 0.2 & 78.6 & 21.2 & Silt \\
\hline 22 & 4 & 2 & $77-79$ & 11.0 & 43.3 & 45.7 & Silty clay \\
\hline 22 & 4 & 2 & $25-27$ & 0.1 & 76.6 & 23.3 & Silt \\
\hline 22 & 4 & 3 & $148-150$ & 3.6 & 46.8 & 49.6 & Silty clay \\
\hline 22 & 4 & 4 & $11-13$ & 4.2 & 46.6 & 49.2 & Silty clay \\
\hline 22 & 4 & 5 & $14-16$ & 1.9 & 73.6 & 24.5 & Clayey silt \\
\hline 22 & 4 & 6 & $20-22$ & 0.5 & 73.0 & 26.5 & Clayey silt \\
\hline 22 & 5 & 1 & 8-10 & 7.1 & 46.6 & 46.3 & Clayey silt \\
\hline 22 & 5 & 2 & $147-148$ & 2.4 & 55.0 & 42.6 & Clayey silt \\
\hline
\end{tabular}


TABLE 2

Leg 3 Summary of the Composition of the Coarse Fraction ${ }^{\text {a }}$

\begin{tabular}{|c|c|c|c|c|c|c|c|c|c|c|c|c|c|c|c|c|}
\hline \multirow[b]{2}{*}{ Hole } & \multirow[b]{2}{*}{ Core } & \multirow[b]{2}{*}{ Section } & \multirow[b]{2}{*}{ 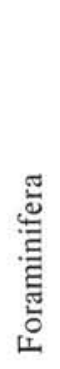 } & \multirow[b]{2}{*}{ 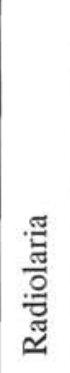 } & \multirow[b]{2}{*}{$\begin{array}{l}\frac{0}{3} \\
\frac{0}{2} \\
\text { के }\end{array}$} & \multirow{2}{*}{ 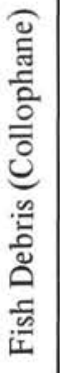 } & \multirow{2}{*}{ 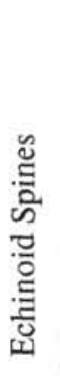 } & \multicolumn{3}{|c|}{ 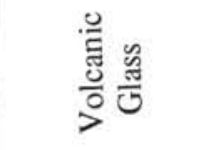 } & \multirow{2}{*}{ 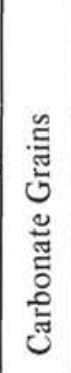 } & \multirow[b]{2}{*}{ 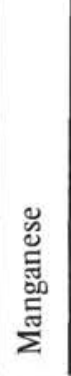 } & \multirow[b]{2}{*}{ 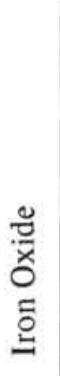 } & \multirow[b]{2}{*}{ 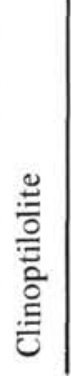 } & \multirow[b]{2}{*}{$\begin{array}{l}\stackrel{\tilde{\Xi}}{\Xi} \\
\frac{\Xi}{\circ}\end{array}$} & \multirow[b]{2}{*}{$\frac{\tilde{\Xi}}{\tilde{0}}$} \\
\hline & & & & & & & & $\frac{\bar{\Xi}}{0}$ & $\frac{3}{\stackrel{0}{J}}$ & 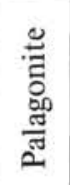 & & & & & & \\
\hline 13 & 1 & $1-6$ & A & & & & & & & & & & & & & \\
\hline 13 & 2 & 1 & A & & & & & & & & & & & & & \\
\hline 13 & 2 & 2 & A & & & $\mathrm{R}$ & & & & & & $\mathrm{R}$ & & & & \\
\hline 13 & 2 & 3 & $\mathrm{~A}$ & & & $\mathrm{R}$ & & $\mathrm{R}$ & $\mathrm{R}$ & & & & & & & \\
\hline 13 & 2 & 4 & $\mathrm{R}$ & $\mathrm{R}$ & & $\mathrm{A}$ & & $\mathrm{A}$ & $\mathrm{C}$ & & & $\mathrm{C}$ & & & & \multirow{4}{*}{$\begin{array}{l}\text { Silica } \\
\text { grains } \\
\text { coated } \\
\text { with iron } \\
\text { oxide R }\end{array}$} \\
\hline 13 & 2 & 5 & $\mathrm{R}$ & $\mathrm{R}$ & & $\mathrm{C}$ & & $\mathrm{R}$ & $\mathrm{R}$ & & & A & & & & \\
\hline 13 & 2 & 5 & & $\mathrm{~A}$ & & & & & & & & & & & & \\
\hline 13 & 2 & 6 & & & & $\mathrm{C}$ & & $\mathrm{R}$ & $\mathrm{R}$ & & & $\mathrm{A}$ & & & & \\
\hline 13 & 3 & $1-6$ & & A & $\mathrm{R}$ & & & & & & & & & & & Diatom C \\
\hline $13 \mathrm{~A}$ & 1 & 1 & & $\mathrm{~A}$ & $\mathrm{C}$ & & & & & & & & & & & Diatom R \\
\hline $13 \mathrm{~A}$ & 2 & 1 & $\mathrm{C}$ & $\mathrm{C}$ & & R & & & & & & & & & & \\
\hline $13 \mathrm{~A}$ & 3 & 1 & $\mathrm{C}$ & $\mathrm{C}$ & & $\mathrm{C}$ & & & & & & $\mathrm{C}$ & & & & \\
\hline $13 \mathrm{~A}$ & 3 & 2 & $\mathrm{~A}$ & & & $\mathrm{C}$ & & & & & & $\mathrm{R}$ & & & & \\
\hline $13 \mathrm{~A}$ & 3 & 3 & $\mathrm{~A}$ & $\mathrm{R}$ & & $\mathrm{C}$ & & $\mathrm{R}$ & & & & $\mathrm{R}$ & & & & \\
\hline $13 \mathrm{~A}$ & 4,5 & 1 & & & & & & & & & $\mathrm{C}$ & $\mathrm{R}$ & & & & Chert A \\
\hline $13 \mathrm{~A}$ & 7 & 1 & & & & $\mathrm{R}$ & & & & & & & & & & Pyrite R \\
\hline 14 & $1 \mathrm{~A}$ & $1-6$ & A & & & $\mathrm{C}$ & $\mathrm{R}$ & & & & & & & & & \\
\hline 14 & 2 & $1-5$ & $\mathrm{~A}$ & & & $\mathrm{R}$ & & & & & & $\mathrm{R}$ & & & & \\
\hline 14 & 3 & $1-6$ & A & & & $\mathrm{R}$ & & & & & & & & & & Ostracod R \\
\hline 14 & 4 & 1 & $\mathrm{C}$ & $\mathrm{C}$ & & & & & & & & & & & & \\
\hline 14 & 5 & $1-6$ & $\mathrm{~A}$ & & & $\mathrm{R}$ & $\mathrm{R}$ & & & & & $\mathrm{R}$ & & & & \\
\hline 14 & 6 & $1-6$ & $\mathrm{~A}$ & & & $\mathrm{R}$ & $\mathrm{R}$ & & & & & & & & & \\
\hline 14 & 7 & $1-6$ & $\mathrm{~A}$ & & & $\mathrm{R}$ & $\mathrm{R}$ & & & & & & & & & \\
\hline 14 & 8 & 1 & $\mathrm{~A}$ & & & $\mathrm{R}$ & $\mathrm{R}$ & & & & & & & & & \\
\hline 14 & 8 & $2-6$ & $\mathrm{~A}$ & & & $\mathrm{R}$ & $\mathrm{R}$ & & & & & $\mathrm{R}$ & & & & \\
\hline 14 & 9 & $2-6$ & $\mathrm{~A}$ & & & $\mathrm{R}$ & $\mathrm{R}$ & & & & & $\mathrm{R}$ & & & & \\
\hline 15 & $1-2$ & $1-6$ & A & & & & & & & & & & & & & \\
\hline 15 & 3 & $1-5$ & $\mathrm{~A}$ & & & & & & & & & & & & & \\
\hline 15 & 3 & 6 & $\mathrm{~A}$ & & & & & & & & & $\mathrm{R}$ & & & & Ostracod R \\
\hline 15 & 4 & $1-6$ & $\mathrm{~A}$ & & & $\mathrm{R}$ & $\mathrm{R}$ & & & & & & $\mathrm{R}$ & & & \\
\hline 15 & 5 & $1-6$ & $\mathrm{~A}$ & & & $\mathrm{R}$ & & & & & & & & & & \\
\hline 15 & 6 & $3,4,6$ & $\mathrm{~A}$ & & & $\mathrm{C}$ & & & & $\mathrm{C}$ & & C & & & & \\
\hline
\end{tabular}


TABLE 2 - Continued

\begin{tabular}{|c|c|c|c|c|c|c|c|c|c|c|c|c|c|c|c|c|}
\hline \multirow[b]{2}{*}{ Hole } & \multirow[b]{2}{*}{ Core } & \multirow[b]{2}{*}{ Section } & \multirow[b]{2}{*}{ 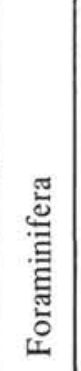 } & \multirow[b]{2}{*}{ 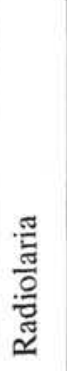 } & \multirow[b]{2}{*}{$\frac{\mathscr{e}}{\tilde{\Xi}}$} & \multirow{2}{*}{ 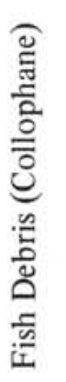 } & \multirow{2}{*}{ 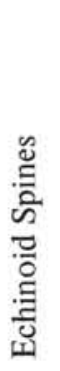 } & \multicolumn{3}{|c|}{ 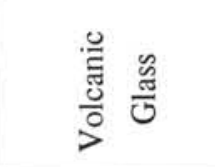 } & \multirow{2}{*}{ 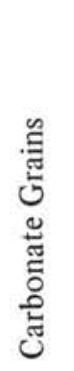 } & \multirow[b]{2}{*}{ 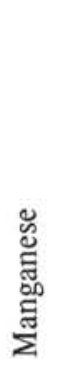 } & \multirow[b]{2}{*}{ 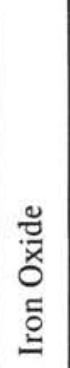 } & \multirow[b]{2}{*}{ 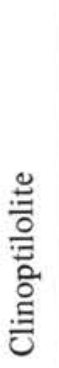 } & \multirow[b]{2}{*}{$\begin{array}{l}\stackrel{\mathscr{\Xi}}{\Xi} \\
\frac{0}{\circ}\end{array}$} & \multirow[b]{2}{*}{ 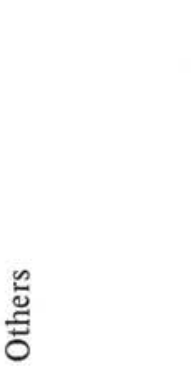 } \\
\hline & & & & & & & & 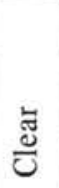 & $\frac{3}{\frac{3}{2}}$ & 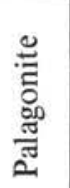 & & & & & & \\
\hline 15 & 7 & $1-6$ & A & & & & & & & & & & & & & \\
\hline 15 & 8 & $1-6$ & $\mathrm{~A}$ & & & & $\mathrm{R}$ & & & & & $\mathrm{R}$ & & & & \\
\hline 15 & 9 & $1-6$ & $\mathrm{~A}$ & & & & $\mathrm{R}$ & & & & & & & & & \\
\hline 16 & 1 & $1,2,4,6$ & A & & & & & & & & & & & & & \\
\hline 16 & 2 & $1-6$ & $\mathrm{~A}$ & & & & & & & & & & & & & \\
\hline 16 & 3 & $1-6$ & $\mathrm{~A}$ & & & & & & & & & & & & & \\
\hline 16 & 4 & $1-6$ & A & & & & & & & & & & & & & \\
\hline 16 & 5 & 1 & $\mathrm{~A}$ & & & & $\mathrm{R}$ & & & & & & & & & Ostracod $\mathrm{R}$ \\
\hline 16 & 5 & $2-6$ & $\mathrm{~A}$ & & & & & & & & & & & & & \\
\hline 16 & 6 & $1-6$ & $\mathrm{~A}$ & & & & $\mathrm{R}$ & & & & & & & & & Ostracod R \\
\hline 16 & 7 & $1,2,4$ & $\mathrm{~A}$ & & & & $\mathrm{R}$ & & & & & & & & & \\
\hline 16 & 7 & 3 & $\mathrm{~A}$ & & & $\mathrm{R}$ & & & & & & & & & & \\
\hline 16 & 7 & 5,6 & $\bar{A}$ & & & & & & & & & & & & & \\
\hline 16 & 8 & $1-6$ & $\mathrm{~A}$ & & & $\mathrm{R}$ & $\mathrm{R}$ & & & & & $\mathrm{R}$ & & & & \\
\hline 16 & 9 & $1-6$ & $\mathrm{~A}$ & & & & $\mathrm{R}$ & & & & & $\mathrm{R}$ & & & & \\
\hline 16 & 10 & $1-6$ & $\mathrm{~A}$ & & & & $\mathrm{R}$ & & & & & $\mathrm{R}$ & & & & Ostracod R \\
\hline 16 & 11 & $1-6$ & $\mathrm{~A}$ & & & & $\mathrm{R}$ & & & & & $\mathrm{R}$ & & & & \\
\hline 17 & 1 & $1-5$ & A & & & & & & & & & & & & & Ostracod R \\
\hline 17 & 2 & $1-4$ & $\mathrm{~A}$ & & & & & & & & & $\mathrm{R}$ & & & & Ostracod R \\
\hline 17 & 2 & 5,6 & $\mathrm{C}$ & $\mathrm{R}$ & $\mathrm{R}$ & & $\mathrm{R}$ & & & & & $\mathrm{R}$ & & & & Diatoms A \\
\hline $17 \mathrm{~A}$ & 1 & $1-6$ & $\mathrm{~A}$ & & & $\mathrm{R}$ & & & & & & & & & & \\
\hline $17 \mathrm{~A}$ & 2 & $1-3$ & $\mathrm{~A}$ & & & $\mathrm{C}$ & $\mathrm{R}$ & & & & & & & & & \\
\hline $17 \mathrm{~A}$ & 2 & 4 & $\mathrm{~A}$ & & & $\mathrm{C}$ & & & & & & & & $\mathrm{R}$ & & \\
\hline $17 \mathrm{~A}$ & 2 & 5,6 & & & & & & & & & & & & & & \\
\hline $17 \mathrm{~A}$ & 3,4 & A11 & $\mathrm{A}$ & & & & $\mathrm{R}$ & & & & & & & & & \\
\hline $17 \mathrm{~B}$ & 1 & $1-6$ & $\mathrm{~A}$ & & & $\mathrm{R}$ & $\mathrm{R}$ & & & & & & & & & Ostracod R \\
\hline 17B & 2 & $1-6$ & $\mathrm{~A}$ & & & $\mathrm{R}$ & $\mathrm{R}$ & & & & & & & & & \\
\hline $17 \mathrm{~B}$ & 3 & $1-6$ & $\mathrm{~A}$ & & & $\mathrm{R}$ & $\mathrm{R}$ & & & & & $\mathrm{R}$ & & & & \\
\hline $17 \mathrm{~B}$ & 4 & & $\mathrm{~A}$ & & & $\mathrm{R}$ & $\mathrm{R}$ & & & & & $\mathrm{R}$ & & & & \\
\hline 18 & 1 & 5 & A & & & & $\mathrm{R}$ & & & & & & & & & \\
\hline 18 & 2 & $1-6$ & $\mathrm{~A}$ & & & $\mathrm{R}$ & $\bar{R}$ & & & & & & & & & Ostracod R \\
\hline
\end{tabular}


TABLE 2 - Continued

\begin{tabular}{|c|c|c|c|c|c|c|c|c|c|c|c|c|c|c|c|c|}
\hline \multirow[b]{2}{*}{ Hole } & \multirow[b]{2}{*}{ Core } & \multirow[b]{2}{*}{ Section } & \multirow[b]{2}{*}{ 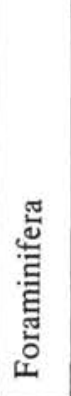 } & \multirow[b]{2}{*}{ 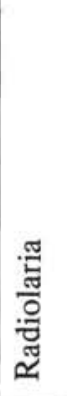 } & \multirow[b]{2}{*}{ 节 } & \multirow{2}{*}{ 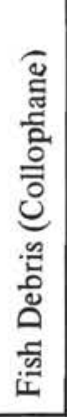 } & \multirow{2}{*}{ 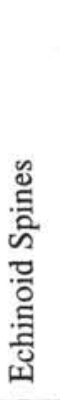 } & \multicolumn{3}{|c|}{ 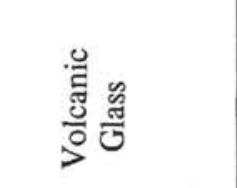 } & \multirow{2}{*}{ 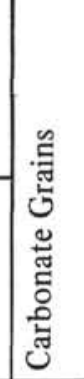 } & \multirow[b]{2}{*}{ 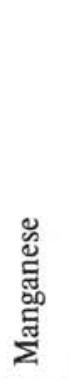 } & \multirow[b]{2}{*}{ 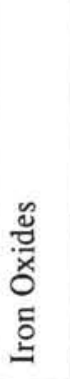 } & \multirow[b]{2}{*}{ 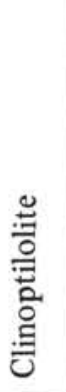 } & \multirow[b]{2}{*}{$\begin{array}{l}\stackrel{\mathscr{\Xi}}{0} \\
\frac{0}{0}\end{array}$} & \multirow[b]{2}{*}{ 点 } \\
\hline & & & & & & & & 䓵 & $\frac{3}{2}$ & 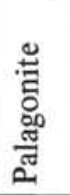 & & & & & & \\
\hline 18 & 3 & $1-6$ & A & & & $\mathrm{R}$ & $\mathrm{R}$ & & & & & $\mathrm{R}$ & & & & Ostracod R \\
\hline 18 & 4 & $1-5$ & A & & & & $\mathrm{R}$ & & & & & & & & & \\
\hline 18 & 5,6 & A11 & $\mathrm{A}$ & & & & $\mathrm{R}$ & 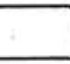 & & & & & & & & \\
\hline 19 & 1 & 1 & A & & & & & & & & & $\mathrm{R}$ & & & & \\
\hline 19 & 1 & $2-6$ & C & & & C & & & & $\mathrm{A}$ & & $\mathrm{C}$ & & $\mathrm{R}$ & & \\
\hline 19 & 2 & $1-6$ & & & & C & & & & $\mathrm{C}$ & & $\mathrm{C}$ & & & & \\
\hline 19 & 3 & $1-6$ & $\mathrm{C}$ & & & $\mathrm{C}$ & $\mathrm{R}$ & & & & & $\mathrm{C}$ & & $\mathrm{R}$ & & Ostracod R \\
\hline 19 & 4 & $1-6$ & $\mathrm{~A}$ & & & $\mathrm{C}$ & $\mathrm{R}$ & & & & & $\mathrm{R}$ & $\mathrm{R}$ & & & Ostracod R \\
\hline 19 & 5 & $1-6$ & A & & & $\mathrm{C}$ & & & & & & $\mathrm{R}$ & $\mathrm{R}$ & & & \\
\hline 19 & 6 & $1-6$ & $\mathrm{~A}$ & & & $\mathrm{C}$ & & & & $\mathrm{R}$ & & $\mathrm{C}$ & & & & \\
\hline 19 & 7 & $1-6$ & $\mathrm{~A}$ & & & $\mathrm{C}$ & & $\mathrm{R}$ & & $\mathrm{C}$ & & $\mathrm{C}$ & & & & \\
\hline 19 & 8,9 & A11 & $\mathrm{A}$ & & & $\mathrm{R}$ & $\mathrm{R}$ & & & $\mathrm{C}$ & & $\mathrm{R}$ & & & & \\
\hline 19 & 10,11 & A11 & $\mathrm{A}$ & & & $\mathrm{R}$ & & & & & & $\mathrm{R}$ & & & & \\
\hline $20 \mathrm{~A}$ & 2 & $1-6$ & A & & & $\mathrm{C}$ & $\mathrm{R}$ & & & & & $\mathrm{R}$ & & & & \\
\hline $20 \mathrm{~B}$ & 1 & $1-6$ & $\mathrm{C}$ & & & $\mathrm{C}$ & & $\mathrm{R}$ & & & & $\mathrm{C}$ & & & & Zeolite R \\
\hline $20 \mathrm{C}$ & 1 & 1 & A & & & & $\mathrm{R}$ & & & & & $\mathrm{R}$ & & & & \\
\hline $20 \mathrm{C}$ & 1 & $2-6$ & $\mathrm{C}$ & & & $\mathrm{C}$ & & & & & & $\mathrm{C}$ & & & & 'Silic. grs. C \\
\hline $20 \mathrm{C}$ & 2 & $1-6$ & $\overline{\mathrm{A}}$ & & & $\mathrm{R}$ & $\mathrm{R}$ & & & & & $\mathrm{R}$ & & & & \\
\hline $20 \mathrm{C}$ & 3 & $1-6$ & $\mathrm{C}$ & & & $\mathrm{C}$ & & $\mathrm{R}$ & & & & $\mathrm{C}$ & & & & Siliceous \\
\hline $20 \mathrm{C}$ & 4 & $1-4$ & $\mathrm{C}$ & & & & & $\mathrm{R}$ & & & & $\mathrm{A}$ & $\mathrm{C}$ & & & grains C \\
\hline $20 \mathrm{C}$ & 5 & $1-4$ & $\mathrm{C}$ & & & $\mathrm{R}$ & & $\mathrm{R}$ & $\mathrm{R}$ & & & $\mathrm{C}$ & $\mathrm{C}$ & & & \\
\hline $20 \mathrm{C}$ & 5 & $5-6$ & $\mathrm{~A}$ & & & & & & & & & & & & & \\
\hline 21 & $1-2$ & Al1 & $\mathrm{A}$ & & & & & & & & & & & & & \\
\hline 21 & 3 & 1 & $\mathrm{~A}$ & & & & & & & & & & & & & \\
\hline 21 & 4 & $1-6$ & A & & & & & & & & & $\mathrm{R}$ & & & & $\begin{array}{l}\text { Ostracod R } \\
\text { Pteropod R } \\
\text { Inoceramus C }\end{array}$ \\
\hline 21 & 5 & $1-6$ & A & & & & $\mathrm{R}$ & & & & & $\mathrm{R}$ & & & & $\begin{array}{l}\text { Ostracod } \mathrm{R} \\
\text { Inoceramus } \mathrm{C}\end{array}$ \\
\hline 21 & 6,7 & All & A & & & & $\mathrm{R}$ & & & & & & & & & $\begin{array}{l}\text { Inoceramus C } \\
\text { Ostracod R }\end{array}$ \\
\hline
\end{tabular}


TABLE 2 - Continued

\begin{tabular}{|c|c|c|c|c|c|c|c|c|c|c|c|c|c|c|c|c|}
\hline \multirow[b]{2}{*}{ Hole } & \multirow[b]{2}{*}{ Core } & \multirow[b]{2}{*}{ Section } & \multirow{2}{*}{ 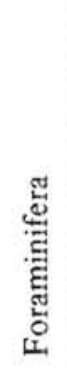 } & \multirow[b]{2}{*}{ 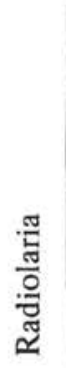 } & \multirow[b]{2}{*}{$\begin{array}{l}\frac{\ddot{\Xi}}{3} \\
\ddot{\ddot{n}} \\
\ddot{n}\end{array}$} & \multirow{2}{*}{ 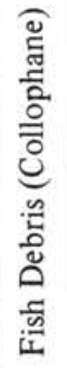 } & \multirow{2}{*}{ 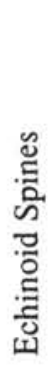 } & \multicolumn{3}{|c|}{$\begin{array}{l}\text { 号 } \\
\frac{\tilde{d}}{0} \\
\frac{\pi}{b}\end{array}$} & \multirow{2}{*}{ 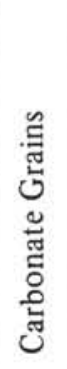 } & \multirow[b]{2}{*}{ 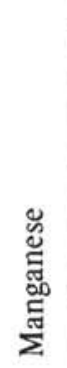 } & \multirow[b]{2}{*}{$\begin{array}{l}u \\
\tilde{u} \\
0 \\
0 \\
0\end{array}$} & \multirow{2}{*}{ 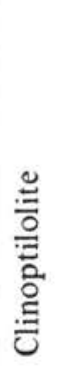 } & \multirow[b]{2}{*}{$\frac{\stackrel{ }{\Xi}}{\frac{0}{\circ}}$} & \multirow[b]{2}{*}{$\begin{array}{l}\text { 岕 } \\
\text { Ẽ }\end{array}$} \\
\hline & & & & & & & & ङ & $\frac{3}{\overline{0}}$ & 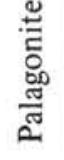 & & & & & & \\
\hline 21 & 8 & $1-6$ & A & & & & $\mathrm{R}$ & & & & & & & & & $\begin{array}{l}\text { Inoceramus C } \\
\text { Ostracod R }\end{array}$ \\
\hline $21 \mathrm{~A}$ & 1 & $1-6$ & $\mathrm{~A}$ & & & & & & & & & & & & & Inoceramus $\mathrm{R}$ \\
\hline $21 \mathrm{~A}$ & 2 & $1-6$ & A & & & $\mathrm{R}$ & & & & & & & & & & \\
\hline $21 \mathrm{~A}$ & 3 & $1-6$ & $\mathrm{~A}$ & & & & & & & & & & & & & Pteropod C \\
\hline 22 & 1,2 & A11 & A & & & & & & & & & & & & & \\
\hline 22 & 3 & $1-5$ & $\mathrm{~A}$ & & $\mathrm{R}$ & & $\mathrm{R}$ & & & & & & & & & \\
\hline 22 & 3 & 6 & $\mathrm{~A}$ & $\mathrm{C}$ & $\mathrm{C}$ & & & & & & & & & & & \\
\hline 22 & 4 & $1-6$ & A & & $\mathrm{R}$ & $\mathrm{R}$ & $\mathrm{R}$ & & & & & & & & & Ostracod R \\
\hline 22 & 5 & 1,2 & $\bar{A}$ & & & & & & & & & & & & & \\
\hline
\end{tabular}

${ }_{\mathrm{A}}^{\mathrm{a}}=\mathrm{ABUNDANT} ; \mathrm{C}=$ COMMON $; \mathrm{R}=\mathrm{RARE}$.

nannoplankton chalk ooze; the high silt content is due to the abundance of nannoplankton. The sand content is low, generally being about 1 to 2 per cent.

\section{Hole 15}

Sediments in the upper part of Hole 15 are silty clay: which show a decrease in sand from 13 to 1 per cent in Cores 1 through 6 and an increase in silt from 18 to 36 per cent in the same cores. In Cores 7 to 9, the silt content increases so that the sediment becomes a clayey silt. All of the sediments here are a nannoplankton chalk ooze.

\section{Hole 16}

The sediments penetrated in Hole 16 range from sandsilt-clay through clayey silt, silty clay to clay. All of the sediments cored in this hole are chalk oozes, and the variation in grain size is due entirely to the fossil content-foraminifera falling in the sand fraction and nannoplankton in the silt fraction.

Core 1 varies from sand-silt-clay with the sand making up 48 per cent of the sediment at the top of the core, to clay at the bottom (with only 9 per cent sand). Core 2 is a sand-silt-clay with 56 per cent clay and approximately equal amounts of sand and silt. Cores 3 through 9 contain silty clays with 12.5 per cent sand in Core 3 and less than 5 per cent in the others. Cores 10 and 11 are either silty clays or clayey silts with sand varying from 3 to 15 per cent.

\section{Hole 17, 17A and 17B}

Hole 17 contains a foraminiferal-nannoplankton chalk ooze in the sandy clay and silty clay size in Core 1 and silty clay size in Core 2 .

Hole $17 \mathrm{~A}$ contains nannoplankton chalk ooze with rare zeolite, and it ranges from silty clay to clayey silt size. In places the silt content is very high, for example, Core 3 with 72.9 per cent silt.

Hole 17B contains nannoplankton chalk ooze in the clayey silt size with a sand content of less than 5 per cent.

It is thought that all variations in the silt percentages are due to the nannoplankton content.

\section{Hole 18}

This hole mainly penetrated nannoplankton chalk ooze in the clayey silt to silty clay size. Core 1 , however, 
TABLE 3

Summary of Leg 3 Grain Size Results Related to Nature of Sediments ${ }^{\text {a }}$

\begin{tabular}{|c|c|c|c|c|}
\hline Hole & Core & Size Classification & Sediment Description & Composition of Coarse Fraction \\
\hline 13 & $1 \& 2$ & Clay & Zeolitic brown clay & Foraminifera $(A)$, collophane $(R)$, volcanic glass $(R)$, mangancse $(R)$, quartz $(R)$ \\
\hline 13 & 3 & Sand-silt-clay or silty clay & Radiolarian ooze & Radiolaria (A), diatom (C), spicules (R) \\
\hline $13 \mathrm{~A}$ & 1 & Silty clay & Radiolarian silty clay and chert & Radiolaria (A), spicules (C), diatom (R) \\
\hline $13 \mathrm{~A}$ & $2 \& 3$ & Clay & Calcareous clay & Foraminifera (C), Radiolaria (C), collophane (C), manganese (R) \\
\hline $13 \mathrm{~A}$ & 4 & Silty clay & Nannofossil chalk ooze & Carbonate $(A)$, chert $(A)$, manganese $(R)$ \\
\hline $13 \mathrm{~A}$ & 5 & Clay & Calcareous dolomitic clay & Carbonate $(\mathrm{A})$, chert $(\mathrm{A})$ \\
\hline $13 \mathrm{~A}$ & 7 & Clay & Red shale & None recovered \\
\hline 14 & 1.9 & Clayey silt or silty clay & Nannofossil chalk ooze & Foraminifera (A), collophane (R), echinoid (R), manganese (R) \\
\hline 15 & $1-6$ & Silty clay & Nannofossil chalk ooze & Foraminifera (A) \\
\hline 15 & 7.9 & Clayey silt or silty clay & Nannofossil chalk ooze & Foraminifera (A) \\
\hline 16 & 1 & Sand-silt-clay, sandy clay, clay & Foraminiferal-nannofossil chalk ooze & Foraminifera (A) \\
\hline 16 & 2 & Sand-silt-clay, sandy clay, silty clay & Foraminiferal-nannofossil chalk ooze & Foraminifera (A) \\
\hline 16 & 3.9 & Silty clay & Nannofossil chalk ooze & Foraminifera (A), echinoid (R) \\
\hline 16 & $10 \& 11$ & Silty clay, clayey silt & Nannofossil chalk ooze & Foraminifera $(A)$, echinoid $(R)$, manganese $(R)$ \\
\hline 17 & 1 & Silty clay, sandy clay, sand-silt-clay & Foraminiferal-nannofossil chalk ooze & Foraminifera (A) \\
\hline 17 & 2 & Silty clay & Foraminiferal-nannofossil chalk ooze & Foraminifera (A), diatoms (A), echinoid (R), manganese (R), Radiolaria (R), spicules (R) \\
\hline $17 \mathrm{~A}$ & 1.4 & Silty clay, clayey silt & Nannofossil chalk ooze & Foraminifera (A), collophane (C), echinoid (R) \\
\hline 17B & 1 & Silty clay, clayey silt & Nannofossil chalk ooze & Foraminifera (A), collophane (R), echinoid (R) \\
\hline $17 \mathrm{~B}$ & $2 \cdot 4$ & Clayey silt & Nannofossil chalk ooze & Foraminifera (A), collophane (R), echinoid (R), manganese (R) \\
\hline 18 & 1 & Clay & Chalk ooze & Foraminifera $(A)$, echinoid $(R)$ \\
\hline 18 & $2 \& 3$ & Clayey silt & Nannofossil chalk ooze & Foraminifera (A), collophane $(R)$, echinoid $(R)$ \\
\hline 18 & $4 \cdot 6$ & Clayey silt or silty clay & Foraminiferal-nannofossil chalk ooze & Foraminifera (A), echinoid ( $R$ ) \\
\hline 19 & $1 \& 2$ & Clay & Zeolitic red clay & Foraminifera (C or lacking), collophane (C), manganese (C), clinoptilolite (R) \\
\hline 19 & $3 \& 4$ & Silty clay & Nannofossil chalk ooze, some zeolite & Foraminifera (A), collophane (C), manganese (C), echinoid (R) \\
\hline 19 & 5.9 & Silty clay, clayey silt & Nannofossil chalk ooze, & Foraminifera (A), collophane (C), manganese (C) \\
\hline 19 & $10-11$ & Silty clay & Nannofossil chalk ooze, & Foraminifera $(A)$, collophane $(R)$, manganese $(R)$ \\
\hline $20 \mathrm{~A}$ & 2 & Silty clay & Nannofossil chalk ooze, & Foraminifera (A), collophane $(C)$, manganese $(R)$, echinoid $(R)$ \\
\hline $20 \mathrm{~B}$ & 1 & Silty clay & $\begin{array}{l}\text { Nannofossil chalk ooze, } \\
\text { and marl }\end{array}$ & Foraminifera (C), volcanic glass (R), collophane (C), manganese (C) \\
\hline $20 \mathrm{C}$ & 1 & Silty clay & Nannofussil marl ooze, some zeolite & Foraminifera $(A)$, manganese $(R)$, echinoid $(R)$ \\
\hline $20 \mathrm{C}$ & 2 & Silty clay, clayey silt & Nannofossil marl-chalk ooze & Foraminifera (A), collophane (R), manganese (R), echinoid (R) \\
\hline $20 \mathrm{C}$ & 3 & Clay, silty clay & Nannofossil marl and zeolitic clay & Foraminifera (A), collophane $(C)$, manganese $(C)$, volcanic glass $(R)$ \\
\hline
\end{tabular}


TABLE 3. (Continued)

\begin{tabular}{lllll}
\hline $20 \mathrm{C}$ & 4 & Silty clay & Nannofossil marl and zcolitic clay & Foraminifera (C), iron oxide (C), manganese (A), volcanic glass (R) \\
$20 \mathrm{C}$ & 5 & Silt, silty clay, clayey silt & Foraminiferal-nannofossil chalk ooze & Foraminifera (C), collophane (R), volcanic glass (R), manganese (C), iron oxide (C) \\
$20 \mathrm{C}$ & 6 & Silty clay & Foraminiferal-nannofossil chalk ooze & Foraminifera (A), collophane (R), volcanic glass (R), mangarese (R). echinoid (R) \\
21 & 1 & Silty and clayey sand, sandy clay & Foraminiferal-nannofossil chalk ooze & Foraminifera (A) \\
21 & 2 & Silty clay, clayey silt & Foraminiferal-nannofossil chalk ooze & Foraminifera (A) \\
21 & $3-7$ & Silty clay, sand-silt-clay & Foraminiferal-nannofossil chalk ooze & Foraminifera (A), Inoceramus (C-Core 4 down), manganese (R-3-5), echinoid (R), pteropod (R) \\
21 & 8 & Sand-silt-clay & Foraminiferal-nannofossil chalk ooze & Foraminifera (A), Inoceramus (C) \\
$21 \mathrm{~A}$ & 1 & Clayey silt, sand-silt-clay & Foraminiferal-nannofossil chalk ooze & Foraminifera (A), Inoceramus (R) \\
$21 \mathrm{~A}$ & 2 & Clayey silt & Foraminiferal-nannofossil chalk ooze & Foraminifera (A) \\
$21 \mathrm{~A}$ & 3 & Sand-silt-clay, silty clay & Foraminiferal-nannofossil chalk ooze & Foraminifera (A), pteropod (C) \\
22 & 1 & Sand-silt-clay, sandy clay, clayey & Foraminiferal-nannofossil chalk ooze & Foraminifera (A) \\
& & sand & & \\
22 & 2 & Sand-silt-clay-silty clay & Foraminiferal-nannofossil chalk ooze & Foraminifera (A) \\
22 & 3 & Clayey silt, silty clay & Foraminiferal-nannofossil chalk ooze & Foraminifera (A), spicules (R), echinoid (R) \\
22 & 4 & Clayey silt, silty clay, silt & Nannofossil chalk ooze & Foraminifera (A), spicules (R). collophane (R), echinoid (C) \\
22 & 5 & Clayey silt & Nannofossil chalk ooze & Foraminifera (A) \\
\hline
\end{tabular}

(A) - Abundant, (C) - Common, (R) - Rare. 
comprised clay with 7.9 per cent sand and 12.5 per cent silt. Cores 2 through 6 are either clayey silt or silty clay with sand showing a progressive increase with depth from 2 per cent in Core 2 to 12 per cent in Core 6.

\section{Hole 19}

Hole 19 penetrated zeolitic clay in Cores 1 and 2. The sand content here is less than 1 per cent, and the silt content is usually 20 to 24 per cent. From Core 3 down there is an increase in the silt content. The sediment is a nannoplankton chalk ooze of silty clay size with some zeolitic nannoplankton material still in Core 3 . Cores 4 through 11 are chalk oozes of either silty clay or clayey silt size with the sand content averaging about 1 to 3 per cent.

\section{Hole 20A, 20B and 20C}

These holes mainly penetrated nannoplankton marl and chalk ooze of silty clay or clayey silt size; in places, zeolitic clay also occurs.

Holes 20A and 20B were in silty clay with less than 1 per cent sand. Hole $20 \mathrm{C}$ consists mainly of silty clay in Cores 1 and 2, with 13 per cent sand near the top of Core 1 but decreasing lower down to less than 1 per cent. In Core 3 clay predominates with about 20 per cent silt and less than 1 per cent sand. Cores 4 and 5 are mainly silty clay with silt reaching as high as 91.6 per cent in one place (Core 5, Section 2). The sand in Core 4 is less than 1 per cent, but increases to 5 per cent in the lower part of Core 5 . Core 6 is all silty clay with less than 5 per cent sand.

\section{Hole 21 and $21 \mathrm{~A}$}

Sediments from Hole 21 are coarser than those from any of the other Leg 3 holes. Silty sand and clayey sand occur in places, though silty clay is still the predominant size grade throughout the hole. The presence of sand grade material is due to the abundance of foraminifera in these nannoplankton chalk oozes.

Core 1 contains as much as 59 per cent sand-with an average of 49.1 per cent sand. The sediment grades from silty sand to sand-silt-clay. Cores 2 through 5 are silty clays with sand less than 20 per cent. Cores 6 through 8 are either silty clay or sand-silt-clay.

Hole $21 \mathrm{~A}$ also penetrates foraminiferal-nannoplankton chalk ooze of clayey silt and sand-silt-clay size in Cores 1 and 2 ; in Core 3 , the sediment becomes finer and changes from sand-silt-clay to silty clay at the base.

\section{Hole 22}

Sediments from Hole 22 are nannoplankton chalk ooze with varying amounts of foraminifera. There is a marked decrease in grain size with depth in this hole because of the decrease in foraminiferal content. Cores 1 and 2 range from clayey sand through sand-silt-clay to silty clay. The sand varies from 15 to nearly 43 per cent. In Core 3 the sand content is about 7 to 10 per cent and the sediment is either silty clay or clayey silt. In Core 4 the sand decreases further and the silt increases to as much as 78.6 per cent. The sediment in Core 5 is of clayey silt size. 